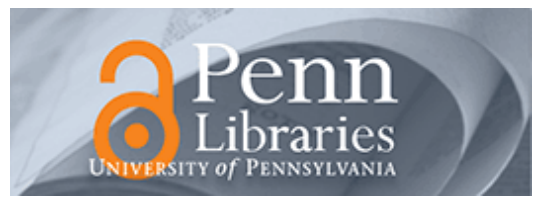

University of Pennsylvania

ScholarlyCommons

Operations, Information and Decisions Papers

Wharton Faculty Research

$5-2009$

\title{
On the Value of Commitment and Availability Guarantees When Selling to Strategic Consumers
}

Xuanming Su

University of Pennsylvania

Fuqiang Zhang

Follow this and additional works at: https://repository.upenn.edu/oid_papers

Part of the Marketing Commons

Recommended Citation (OVERRIDE)

availability guarantee, commitment, product availability, search costs, stockouts, newsvendor, rational expectations

This paper is posted at ScholarlyCommons. https://repository.upenn.edu/oid_papers/156

For more information, please contact repository@pobox.upenn.edu. 


\title{
On the Value of Commitment and Availability Guarantees When Selling to Strategic Consumers
}

\author{
Abstract \\ This paper studies the role of product availability in attracting consumer demand. We start with a \\ newsvendor model, but additionally assume that stockouts are costly to consumers. The seller sets an \\ observable price and an unobservable stocking quantity. Consumers anticipate the likelihood of \\ stockouts and determine whether to visit the seller. We characterize the rational expectations equilibrium \\ in this game. We propose two strategies that the seller can use to improve profits: (i) commitment (i.e., \\ the seller, ex ante, commits to a particular quantity) and (ii) availability guarantees (i.e., the seller \\ promises to compensate consumers, ex post, if the product is out of stock). Interestingly, the seller has \\ an incentive to overcompensate consumers during stockouts, relative to the first-best benchmark under \\ which social welfare is maximized. We find that first-best outcomes do not arise in equilibrium, but can be \\ supported when the seller uses a combination of commitment and availability guarantees. Finally, we \\ examine the robustness of these conclusions by extending our analysis to incorporate dynamic learning, \\ multiple products, and consumer heterogeneity. \\ Disciplines \\ Marketing
}




\section{On the Value of Commitment and Availability Guarantees When Selling to Strategic Consumers}

Article in Management Science · May 2009

Impact Factor: $2.48 \cdot$ DOI: $10.2139 /$ ssrn.1010269 $\cdot$ Source: DBLP

CITATIONS

55

2 authors:

\section{Xuanming Su}

University of Pennsylvania

27 PUBLICATIONS 1,021 CITATIONS

SEE PROFILE
READS

47
Fuqiang Zhang

Washington University in St. Louis

33 PUBLICATIONS 1,517 CITATIONS

SEE PROFILE 


\title{
On the Value of Commitment and Availability Guarantees When Selling to Strategic Consumers
}

\author{
Xuanming $\mathrm{Su}$ \\ Fuqiang Zhang \\ xuanming@haas.berkeley.edu \\ fzhang22@wustl.edu \\ faculty.haas.berkeley.edu/xuanming/ \\ Olin Business School \\ The Haas School of Business \\ Washington University in St. Louis \\ University of California, Berkeley \\ St. Louis, MO63130 \\ Berkeley, CA 94720 \\ June, 2007; revised December, 2007, July, 2008
}

\begin{abstract}
This paper studies the role of product availability in attracting consumer demand. We start with a newsvendor model, but additionally assume that stockouts are costly to consumers. The seller sets an observable price and an unobservable stocking quantity. Consumers anticipate the likelihood of stockouts and determine whether to visit the seller. We characterize the rational expectations (RE) equilibrium in this game. We propose two strategies that the seller can use to improve profits: (i) commitment (i.e., the seller, ex-ante, commits to a particular quantity), and (ii) availability guarantees (i.e., the seller promises to compensate consumers, ex-post, if the product is out of stock). Interestingly, the seller has an incentive to over-compensate consumers during stockouts, relative to the first-best benchmark under which social welfare is maximized. We find that first-best outcomes do not arise in equilibrium, but can be supported when the seller uses a combination of commitment and availability guarantees. Finally, we examine the robustness of these conclusions by extending our analysis to incorporate dynamic learning, multiple products, and consumer heterogeneity.
\end{abstract}

Keywords: availability guarantee, commitment, product availability, search costs, stockouts, newsvendor, rational expectations 


\section{Introduction}

Product availability is an essential ingredient for turning demand into sales. More importantly, product availability is indispensable for attracting demand in the first place. Consumers would not patronize a firm without some form of assurance that they can find what they are looking for. Despite technological and managerial advances, stockout situations are common phenomenon and product availability remains a key issue in marketing and operations (Balachander and Farquhar, 1994; Anderson, Fitzsimons and Simester, 2006). Many industry studies have found that about 8 percent of retail items are out of stock at any one time, and the percentage can be much higher for advertised items (e.g., Andersen Consulting, 1996, and Gruen and Corsten, 2008). Since stockouts may ruin the shopping experience, the consumer is less willing to make necessary up-front investments to visit the store, which is thus less likely to maintain satisfactory levels of availability. This creates a chicken-or-egg issue, which may spiral toward market breakdown. In this paper, we study the role of product availability in shaping market interactions between the firm and its consumers.

The video rental industry provides an interesting example (see Dana, 2000). According to a Time Warner survey, about $20 \%$ of customers were not able to rent their first-choice movie title on a typical visit. Some customers who find their preferred movie stocked out may leave the store empty-handed, and the possibility of wasted trips may discourage future visits. To address this problem, Blockbuster initiated the "Go Home Happy" program, which guarantees availability of selected new releases in the form of a rain check for the same movie if it was unavailable (see Furman, 1998, and Dana and Spier, 2001). Consumers rated the availability guarantees as a highly desirable attribute of a video rental store (see Video Software Dealers Association, 1999).

The out-of-stock problem has also attracted much attention in the consumer goods industry. In a comprehensive study covering 32 product categories in 661 retail outlets across 29 countries, Gruen and Corsten (2002) show that overall out-of-stock rates are consistently in the range of 5 to 10 percent, and often exceed 10 percent for items on promotion. Fitzsimons (2000) finds that consumers exposed to a stockout are substantially less likely to return to the same store on their next shopping trip. Although stockouts lead to decreased customer satisfaction, firms can adopt policies to mitigate their effects. For example, through consumer surveys, Verhoef and Sloot (2005) find that consumers appreciate discount coupons, rain checks, and additional services, such as home delivery, for out-of-stock items.

The problem of product unavailability is not limited to traditional brick-and-mortar retail firms. 
In the case of mail-order catalog companies, customers even report stockouts as their chief complaint (see Fitzsimons, 2000, and Consumer Reports, 1987). This is not surprising as catalog companies, particularly specialty merchandisers, typically stock less than $80 \%$ of their advertised items (Balachander and Farquhar, 1994). It is thus common practice in the catalog industry to offer financial compensation, such as price discounts or reduced shipping charges, to customers ordering out-of-stock items (Anderson, Fitzsimons and Simester, 2006). Similarly, some online retailers offer monetary incentives for customers to place a backorder on currently unavailable items (Breugelmans, Campo and Gijsbrechts, 2006; Bhargava, Sun and Xu, 2006). To the extent that this strategy is effective in sustaining consumer demand, some e-tailers have even moved to a "stockless" mode of operation, relying entirely on using discounts to compensate consumers for stockout related costs (Sun, Ryan and Shin, 2008).

Consumers are concerned about product availability because stockouts are costly to them. When a desired item is out of stock, consumers may face increased decision making cost (e.g., of having to choose a substitute), increased transaction cost (e.g., from going to another store to purchase the item), as well as other inconveniences (e.g., when delaying the purchase). In addition, consumers often invest time and energy into their shopping trips. These costs are sunk and not recoverable. In many cases, these sunk costs can be quite substantial. When shopping for electronics, toys, or video rentals, customers typically travel to the store and locate their items on the shelf. Some consumers also spend time checking product reviews beforehand. For fashion apparel and footwear, customers often need to spend time trying on different sizes or styles before finalizing their selection (if available). For specialty items such as fragrance and skincare, customers need to evaluate the product at the store before making a purchase decision. Even for online purchases, the frictional costs for navigating through webpages and submitting orders could be substantial (see Hann and Terwiesch, 2003). All these effort will be in vain if they culminate in an out-of-stock experience. This discussion brings us to the main premise of this paper: since stockouts are costly to consumers, product availability has a direct effect on demand.

Our model has two main elements: demand uncertainty and consumer costs. Demand uncertainty is important because it is the potential mismatch between supply and demand that generates stockouts. Consumer costs are also important because they are the reason why consumers are concerned about stockouts in the first place. In our model, there is a newsvendor seller who makes pricing and inventory decisions. Consumers choose whether to visit the seller and incur sunk costs if they do. The seller's objective is to maximize expected profits, and consumers wish to maximize 
individual utility. Following our earlier work, Su and Zhang (2008), we adopt the rational expectations (RE) paradigm. Specifically, consumers form rational expectations of product availability and make their patronage decisions accordingly; similarly, the seller forms rational expectations of consumer behavior and acts accordingly. Using this setup, we characterize consumer behavior as well as optimal prices and inventory levels in the RE equilibrium. We also study the case where the seller can commit to some (presumably high) service level. In addition, we consider availability guarantees - that is, when the product is out of stock, the seller compensates consumers through monetary payments, store credit, or other forms of goodwill services. Throughout our analysis, we use the first best (under which social efficiency is maximized) as a benchmark to evaluate market outcomes.

Let us now summarize our three main findings. First, we show that the ability to commit enhances the seller's profits. The intuition is as follows. Normally, consumers make patronage decisions based on their expectations of product availability. In contrast, when the seller commits to a particular service level, he is effectively taking the lead in this game and steering the equilibrium toward higher expectations and increased product availability. However, unless credibility is sustained by some external commitment device (e.g., the seller's reputation concerns, consumer laws or a third-party endorsement), consumers may not trust the seller's claims. This issue of credibility suggests that commitment may not always be feasible in practice.

Our second major finding is that availability guarantees are useful contractual mechanisms. By artificially inflating the seller's costs of stockouts, such guarantees help the seller commit to higher service levels, which attracts customer patronage and increases willingness to pay. In fact, with availability guarantees, the seller can attain or even surpass profit levels under commitment. This finding suggests that availability guarantees can serve as a replacement for a commitment device. In our analysis, we consider various types of availability guarantees. When a product is out of stock, the seller may offer monetary compensation, or alternatives (such as store credit or discount coupons) through which the seller may exploit cost savings. That is, the cost of providing stockout compensation may be less than its value perceived by consumers. We find that such cost savings provide an additional avenue for the seller to improve profits. However, this is a double-edged sword as it creates incentives for the seller to generate market inefficiencies. Specifically, the seller tends to over-compensate consumers during stockouts, because such an arrangement allows him to realize a higher-then-efficient price level.

Finally, we discuss the welfare implications of our results. As a benchmark for comparison, 
we study the first-best market outcomes under which social welfare is maximized. In general, we discover that efficiency is not attained in equilibrium. In most cases, the seller tends to set prices too high, set quantities too low, and offer excessively generous availability guarantees. Nevertheless, we find that the first-best can indeed be supported in equilibrium using a combination of commitment and availability guarantees. This suggests that when addressing the interplay between product availability and consumer behavior, both ex ante and ex post considerations are important. The seller should commit to high service level ex ante, and at the same time should insure consumers against ex post losses.

The remainder of this paper is organized as follows. Section 2 reviews the literature. In Section 3 , we set up our basic model and characterize the seller's price, quantity, and profits in the RE equilibrium. In Section 4, we study the value of the ability to commit by comparing this to our basic model. In Section 5, we analyze availability guarantees: we first consider offering monetary compensation to consumers facing stockouts, and then proceed to consider using more general forms of compensation. In Section 6, we characterize the first-best and show that it can be attained using a combination of service level commitment and availability guarantees. Section 7 presents several extensions of the basic model, and shows that our main results remain unchanged. Finally, we offer concluding remarks in Section 8. All proofs are presented in the Appendix.

\section{Literature Review}

Most closely related to our work is the stream of literature on using product availability as a strategic lever to attract or induce demand. Dana and Petruzzi (2001) consider a newsvendor setup, similar to ours, in which demand depends on the firm's price and inventory. In the model, consumers forego an outside option when she visits the firm before knowing whether or not the product will be available. Alexandrov and Lariviere (2007) recently analyzed a related model in the context of restaurants, where walk-in customers may be declined a seat if the restaurant is full. In these settings, higher inventory levels and higher capacity attract consumers and increases their willingness to pay. Here, a common assumption is that prices and inventory levels are observed by consumers. In a competitive setting, Deneckere and Peck (1995) study firms' pricing and inventory decisions when demand is stochastic. Similar to newsvendor models, the aggregate demand is random and each consumer chooses to visit one (and only one) firm based on each firm's price and inventory level, which are assumed to be publicly observable, similar to the papers above. The 
authors demonstrate that unless the number of firms is large, a pure strategy equilibrium may fail to exist because firms have an incentive to undercut each other's prices, similar to models of Bertrand competition. Dana (2001) extends this analysis to the case of observable prices but unobservable inventory levels. He shows that in this case, prices tend to be positively related to inventory levels. Under Bertrand timing (where firms choose prices first), higher prices induce firms to hold higher inventories, whereas under Cournot timing (where firms choose inventories first), higher prices can be used to "signal" higher availability. In our present work, we consider both cases: unobservable inventory (in the basic model in Section 3) as well as observable inventory (which falls under the case with seller commitment in Section 4). Further, we study the impact of availability guarantees on equilibrium outcomes.

This research follows recent interest in strategic customer behavior in the operations management community. By our terminology, customers are strategic in the sense that they are active decision-makers and respond strategically to underlying economic mechanisms (such as availability guarantees). In our current setting, high inventory levels stimulate demand by providing an assurance of availability. However, there are also situations in which inventory has the opposite effect on customer demand. In a companion paper, Su and Zhang (2008), we consider customers who may strategically wait for markdowns at the end of the selling season, and we study the implications of such behavior on supply chain performance. In that setting, a high inventory level deflates regularseason demand because it increases the firm's likelihood of having leftover units and thus increases the consumer's chances of obtaining the product on sale at the end of the selling season. Cachon and Swinney (2007) also study consumers' strategic waiting for sales. They demonstrate that the value of quick response strategies is much higher in the presence of strategic consumers. In another paper, Lai, Debo, and Sycara (2007) examine the effectiveness of using price matching policies to discourage strategic waiting for sales. Here, the seller agrees to reimburse the price difference to consumers if the items that they buy are subsequently marked down. Next, Jerath, Netessine, and Veeraraghavan (2007) demonstrate that, when facing strategic customers, opaque selling (i.e., obscuring from customers the exact product specifications, such as departure times in the context of airlines) can enhance seller profits. In the same spirit as our work, these papers are based on the rational expectations paradigm (see Muth, 1961, and Stokey, 1981). Consumers make patronage or purchase decisions based on rational expectations of product availability, and the seller makes pricing and inventory decisions based on rational expectations of consumer behavior.

There is another stream of work that focuses on the dynamics of consumer forward-looking 
behavior. This began with the durable goods monopoly literature in economics. The seminal Coase conjecture (1972) states that when consumers rationally expect future price decreases, the monopoly is forced to price at marginal cost. Representative studies include Stokey (1979, 1981), Bulow (1982), and Besanko and Winston (1990). However, all these papers do not consider inventory constraints. In the operations literature, Aviv and Pazgal (2008) are the first to analyze the dynamic pricing problem with strategic customers and inventory constraints. Liu and van Ryzin (2008) offer a different perspective by capturing this scenario using a quantity-rationing model rather than a pricing model. Su (2007) considers a mixture of strategic and myopic consumers and demonstrates that this has a significant impact on the optimal pricing policy. Yin, Aviv, Pazgal and Tang (2007) study the effect of inventory information on consumer purchase behavior; this is also one of the main themes of our present work. For a comprehensive review of the literature on dynamic pricing with strategic customers, readers are referred to Shen and Su (2007).

\section{Basic Model}

We begin by describing our basic model. There is a newsvendor seller who announces the price $p$ and stocks a certain quantity $q$ at unit cost $c$. Leftover units have zero salvage value. The market demand $X$ is random and follows distribution $F$. This market demand can be interpreted as a mass of infinitesimal consumers. Assume $F$ has an increasing failure rate, which is satisfied by most commonly used distribution functions. Each individual consumer has valuation $v$ for the product and faces a search cost of $h$ (e.g., transportation cost, shopping time, etc) in order to visit the seller. If the product is out of stock, this sunk cost is not recoverable and the consumer incurs an additional inconvenience cost of $h^{\prime}$ (e.g., from foregoing or delaying the purchase). Therefore, consumers who visit the seller face three possible outcomes: they receive payoff $v-p-h$ if the product is available or payoff $-\left(h+h^{\prime}\right)$ if the product is sold out; consumers who do not visit the firm receive zero payoff. Without loss of generality, we assume $h^{\prime}=0$; otherwise, we use $v+h^{\prime}$ in place of $v$ and $h+h^{\prime}$ in place of $h$ above. We initially assume that consumers are homogeneous, so all consumers who visit the seller are equally likely to receive the product in the event of scarcity. For a nontrivial outcome to exist, we assume $0<h<v-c$. Define $E$ as the expectation operation and $\wedge$ as the minimum operation, i.e., $x \wedge y=\min (x, y)$. We use an upper bar to denote the complement function, e.g., $\bar{F}=1-F$.

In this model, the seller chooses price $p$ and quantity $q$ to maximize expected profit. The price 
is publicly known but the quantity is not observed by individual consumers. On the other hand, consumers choose a reservation price $r$ to maximize individual expected payoff. This reservation price provides a decision threshold: accordingly, consumers will visit the seller if and only if the seller's price $p$ does not exceed this threshold $r$.

To study this strategic interaction, we shall use the notion of a rational expectations (RE) equilibrium. This equilibrium concept has also been used on other operations models (see Stokey, 1981, Cachon and Swinney, 2007, Su and Zhang, 2008). We describe the equilibrium concept in our context by separately considering the consumer's and the seller's decision problems.

Consider first the typical consumer's problem. Intuitively, the consumer's reservation price should depend on product availability: the more likely it is to obtain the product after visiting the seller, the higher the willingness to pay before agreeing to visit the seller in the first place. However, the consumer is unable to ascertain the probability of finding the product in stock because this probability depends on the seller's quantity $q$, which is not observable. Nevertheless, the consumer may possess some beliefs over this probability of availability. Suppose that the consumer expects to obtain the product with probability $\xi_{\text {prob }}$ if she visits the seller. Under these beliefs, the consumer expects to earn surplus $(v-p) \xi_{\text {prob }}-h$ from visiting the seller (and zero otherwise). This implies that the consumer's reservation price $r$ satisfies

$$
(v-r) \xi_{\text {prob }}=h
$$

Next, we consider the seller's problem. To maximize expected profit, the seller should set price equal to consumers' reservation price, since this is the highest price at which consumers are willing to visit the seller. However, the seller does not know consumers' reservation prices. Similarly as before, we endow the seller with beliefs $\xi_{r}$ over consumers' reservation prices. Given these beliefs, the seller chooses price $p=\xi_{r}$ and stocks the critical fractile quantity $q=\bar{F}^{-1}(c / p)$ to maximize the newsvendor profit function $\Pi(q)=p E(X \wedge q)-c q$.

Given players' rational expectations, the strategic game between the seller and the consumers decomposes into two separate decision problems (a binary choice problem for consumers and a newsvendor problem for the seller), both of which are easy to solve and have unique solutions. Moreover, each decision problem does not require information about the other side of the market. For example, given beliefs $\xi_{\text {prob }}$, consumers do not need to know the seller's cost $c$ or demand distribution $F$. Similarly, given beliefs $\xi_{r}$, the seller does not need to know the consumer's valuation $v$ or search cost $h$. Therefore, once all players have their beliefs in place, they will naturally settle 
into their optimal actions.

In equilibrium, beliefs must be consistent with outcomes. In other words, the seller's beliefs $\xi_{r}$ must coincide with consumers' reservation price $r$, and consumers' beliefs over availability probability $\xi_{\text {prob }}$ must agree with the actual in-stock probability corresponding to the seller's chosen quantity $q$. In fact, this probability is given by $A(q) \equiv \frac{E(X \wedge q)}{E(X)}$. The argument proceeds as follows, and is due to Deneckere and Peck (1995) and further explored by Dana (2001). Notice that the product is available with probability $\frac{x \wedge q}{x}$, when there are $x$ consumers in the market. Since the market size is uncertain, conditioning on his own presence in the market, an individual consumer's posterior demand density is $g(x)=x f(x) / E(X)$ (where $f(x)$ is the prior demand density). Now, integrating over this posterior demand density, the availability probability becomes

$$
\int \frac{(x \wedge q)}{x} g(x) d x=\int \frac{(x \wedge q) f(x)}{E(X)} d x=A(q) .
$$

Summarizing the preceding discussion, we have the following definition of a RE equilibrium. ${ }^{1}$

Definition 1 A rational expectations (RE) equilibrium consists of $\left(p, q, r, \xi_{\text {prob }}, \xi_{r}\right)$ satisfying:

(i) $r=v-h / \xi_{\text {prob }}$,

(ii) $\bar{F}(q)=c / p, p=\xi_{r}$

(iii) $\xi_{\text {prob }}=A(q), \xi_{r}=r$.

The three conditions (i), (ii), (iii) follow respectively from the consumer's optimal decision $r$ given beliefs $\xi_{\text {prob }}$, the seller's optimal decisions $p, q$ given beliefs $\xi_{r}$, and consistency conditions. Here, we consider the scenario where all players are endowed with accurate beliefs of market outcomes (i.e., rational expectations). The assumption that customers possess fully rational expectations has been invoked in other operations settings (see Liu and van Ryzin, 2008 for a capacity rationing model and Hassin and Haviv, 2003 for queue-joining models). In Section 7.1, we extend our model to a dynamic setting and show that players can form rational expectations through adaptive learning (cf. Liu and van Ryzin, 2007).

\footnotetext{
${ }^{1}$ In Definition 1, we have implicitly restricted attention to equilibria in which the market is functional; in other words, the seller stocks nonzero quantities and there are some consumers visiting the seller. Notice that there will always be another non-participatory equilibrium. Here, the trivial outcome is that the seller selects $q=0$ and thus no consumer visits the seller. In fact, this outcome can always be supported by beliefs $\xi_{\text {prob }}=\xi_{r}=0$. These are self-fulfilling prophecies that induce market failure solely because market participants do not believe it will work in the first place. When referring to an "RE equilibrium" in the rest of this paper, we are referring to a participatory equilibrium. While there is always a non-participatory equilibrium, a participatory equilibrium may not exist.
} 
The following proposition characterizes the RE equilibrium and provides conditions under which it exists.

Proposition 1 There exists some $\bar{h}<v-c$ such that a $R E$ equilibrium exists if and only if $h<\bar{h}$, where $\bar{h}$ increases in $v$ and decreases in $c$. In any RE equilibrium, the seller's price $p^{*}$ and quantity $q^{*}$ satisfy

$$
\begin{aligned}
\bar{F}\left(q^{*}\right) & =\frac{c}{p^{*}}, \\
\left(v-p^{*}\right) A\left(q^{*}\right) & =h .
\end{aligned}
$$

Moreover, the seller's equilibrium profit is

$$
\Pi^{*}=v E\left(X \wedge q^{*}\right)-c q^{*}-h E(X) .
$$

This lemma implies that the market may break down when the search cost is above a certain threshold value $\bar{h}$. To avoid trivial outcomes, we impose the following assumption hereafter unless otherwise mentioned. This assumption ensures that the search cost $h$ is below the threshold value, and guarantees the existence of a RE equilibrium.

Assumption 1 Consumer search cost $h$ satisfies $h<\bar{h}$.

\section{Value of Commitment}

The RE equilibrium derived above assumes that consumers rely entirely on their rational expectations to draw inferences about product availability. In our model, suppose that the seller additionally makes explicit announcements regarding product availability. For example, the seller may announce to the market that he intends to stock quantity $\widetilde{q}$ and maintain the fill rate $A(\widetilde{q})$. How should consumers view these announcements $\widetilde{q}$ ?

In our model above, consumers should simply ignore the seller's claims. This is because the seller has an incentive to convince consumers that service levels will be high, since higher availability implies higher willingness-to-pay. Therefore, the seller tends to exaggerate the announced quantity $\widetilde{q}$, which is thus not credible. Consumers rely on their rational expectations, and the RE equilibrium above characterizes market outcomes.

Now, suppose there is some external commitment device that requires the seller to keep his word. That is, the seller's announced quantity $\widetilde{q}$ must coincide with the true quantity $q$. The 
commitment device can be interpreted in several ways. For example, consumers may be able to directly monitor the seller's inventory and verify that the announcement $\widetilde{q}=q$ is accurate. Alternatively, when stocking quantities and fill rates are verifiable ex post, the seller may be averse to misrepresentation due to reputation concerns. Also, there may be legal institutions that enforce the seller's truth-telling constraint.

We next analyze the market outcomes with seller commitment. In any equilibrium, consumers' beliefs $\xi_{\text {prob }}$ must be consistent with the actual availability probability $A(q)$, which is determined by the seller's committed quantity $q$. Thus they are willing to visit the seller as long as price does not exceed the reservation price $r=v-h / A(q)$. Corresponding to quantity $q$, it is optimal for the seller to charge price $p(q)=v-h / A(q)$. The seller's optimal quantity then follows from maximizing the following profit function

$$
\begin{aligned}
\Pi_{C}(q) & =p(q) E(X \wedge q)-c q \\
& =\left(v-\frac{h}{A(q)}\right) E(X \wedge q)-c q \\
& =v E(X \wedge q)-c q-h E(X) .
\end{aligned}
$$

Here, the subscript $\mathrm{C}$ refers to the case with seller commitment. The following proposition characterizes market outcomes under seller commitment.

Proposition 2 Suppose that the seller can commit to quantity $q$. There exists an $\bar{h}_{C}>\bar{h}$ such that an equilibrium exists if and only if $h<\bar{h}_{C}$. In equilibrium, the seller's price $p_{C}^{*}$ and quantity $q_{C}^{*}$ satisfy

$$
\begin{aligned}
\bar{F}\left(q_{C}^{*}\right) & =\frac{c}{v}, \\
\left(v-p_{C}^{*}\right) A\left(q_{C}^{*}\right) & =h .
\end{aligned}
$$

Moreover, the seller's equilibrium profit is

$$
\Pi_{C}^{*}=v E\left(X \wedge q_{C}^{*}\right)-c q_{C}^{*}-h E(X) .
$$

A direct comparison of Propositions 1 and 2 reveals that the seller's commitment power changes market outcomes dramatically. In fact, the next proposition demonstrates that commitment power is valuable to the seller. Under commitment, consumers are better able to assess their chances of securing the product if they do visit the seller (at a cost $h$ ). All else equal, this encourages consumer visits and increases their willingness to pay. This effect in turn increases profit margins 
and leads the seller to stock higher quantities, thus providing higher service levels in the first place. Therefore, both price and quantity are increased, and the overall effect on seller profits is positive.

Proposition 3 The equilibrium price, quantity, and seller profits are all higher with commitment. In other words, $p_{C}^{*}>p^{*}, q_{C}^{*}>q^{*}$, and $\Pi_{C}^{*}>\Pi^{*}$.

The improved outcome is a direct consequence of commitment and can not be sustained when the seller does not commit to quantity $q$. Consider the following hypothetical scenario without seller commitment. Suppose the seller sets price $p_{C}^{*}$ and stocks quantity $q_{C}^{*}$, and consumers are willing to visit the seller. However, once consumers are willing to visit the seller at price $p_{C}^{*}$, the seller's profit function effectively becomes $\Pi(q)=p_{C}^{*} E(X \wedge q)-c q$ (i.e., with a fixed price), and the optimal stocking quantity is $\bar{F}^{-1}\left(c / p_{C}^{*}\right)$, which is less than $q_{C}^{*}=\bar{F}^{-1}(c / v)$. Therefore, when the seller does not commit to $q_{C}^{*}$, he has an incentive to decrease quantity below $q_{C}^{*}$ once consumers are willing to pay $p_{C}^{*}$. This implies that consumers will not pay price $p_{C}^{*}$ in the first place, and the outcome with price $p_{C}^{*}$ and quantity $q_{C}^{*}$ is not sustainable without seller commitment.

\section{$5 \quad$ Availability Guarantees}

Although we have seen that commitment power is valuable to the seller, a viable commitment device may not exist in practice. In such cases, is it possible for the seller to do better than the RE equilibrium outcome of Section 3? In particular, using the commitment case as a benchmark, is it possible to attain or even surpass the profit level $\Pi_{C}^{*}$ ? In this section, we show that availability guarantees can be used to stimulate demand and improve seller profits. We use the term "availability guarantee" to refer to mechanisms in which the seller promises to compensate consumers in the event of a stockout.

In practice, firms may offer availability guarantees that come in many different forms. For example, if a particular item is out of stock, retailers may offer to ship it to the consumer at no extra charge. This may be implemented through transshipment between retail locations, or from a central warehouse. As another example, consider movie rental companies. When a particular movie is stocked out, the company may offer a free rental on the same movie during a future visit. Finally, the seller may offer gift cards or discount coupons to placate consumers who do not find their desired items in stock. See Dong and Rudi (2004), Dana (2000), and Bhargava, Sun and Xu (2006) for some anecdotal evidence of various forms of availability guarantees. Of course, the seller 
may also simply offer monetary compensation. In this section, we describe a general framework to analyze such availability guarantees.

We model availability guarantees as follows. Suppose the seller agrees to compensate customers who experience stockouts. Such compensation may take different forms as described above. Let $u$ denote the consumer's utility from receiving the compensation, and let $w$ denote the seller's cost of compensating one such consumer. We emphasize that these payoffs and costs are relevant only when stockouts occur. In our model, each utility-cost pair $(u, w)$ represents a particular guarantee format. Observe that when the utility from stockout compensation $u$ exceeds consumer search cost $h$, there may be a moral hazard problem in which consumers without any intention of buying still visit the seller with hopes of being compensated. Fortunately, firms will typically never offer guarantees that are inappropriately generous. Therefore, throughout the rest of our analysis, we assume that the seller knows that $u \leq h$ is satisfied. A sufficient (but not necessary) condition for this assumption is when the seller has an accurate estimate of $h$. In general, we distinguish between three different classes of availability guarantees: monetary compensation (where the seller pays cash to customers facing stock-outs, so $w=u$ ), efficient guarantees (with $w \leq u$ ), and inefficient guarantees (with $w \geq u$ ). The choice between different formats for availability guarantees will be explored later. Now, we first focus on a fixed type of guarantee $(u, w)$ and investigate the RE equilibrium outcome under this guarantee.

Following the same reasoning as before, we see that, given beliefs $\xi_{\text {prob }}$, consumers' reservation prices $r$ satisfy

$$
(v-r) \xi_{\text {prob }}+u\left(1-\xi_{\text {prob }}\right)=h .
$$

On the other hand, considering the seller's cost and assuming that consumers are willing to buy, the seller's profit function is

$$
\Pi(p, q)=p E(X \wedge q)-c q-w[E(X)-E(X \wedge q)]
$$

so the seller stocks the critical fractile quantity $q=\bar{F}^{-1}\left(\frac{c}{p+w}\right)$. Together with consistency conditions, we can then characterize the resulting RE equilibrium corresponding to the $(u, w)$ guarantee. The equilibrium conditions are given in the next result. The subscript $G$ refers to availability guarantees.

Proposition 4 With availability guarantee of type $(u, w)$, the $R E$ equilibrium $\left(p_{G}^{*}, q_{G}^{*}\right)$ is charac- 
terized by

$$
\begin{aligned}
\bar{F}\left(q_{G}^{*}\right) & =\frac{c}{p_{G}^{*}+w}, \\
\left(v-p_{G}^{*}\right) A\left(q_{G}^{*}\right)+u \bar{A}\left(q_{G}^{*}\right) & =h .
\end{aligned}
$$

Moreover, the seller's equilibrium profit is

$$
\Pi_{G}^{*}=v E\left(X \wedge q_{G}^{*}\right)-c q_{G}^{*}-h E(X)+(u-w)\left[E(X)-E\left(X \wedge q_{G}^{*}\right)\right] .
$$

When $w=u$, there is a threshold value $\bar{h}<\bar{h}_{G} \leq \bar{h}_{C}$ such that an equilibrium exists if and only if $h<\bar{h}_{G}$.

Note that availability guarantees may raise the threshold search cost for the existence of equilibrium, i.e., $\bar{h}_{G}>\bar{h}$ for the benchmark case $w=u$. Clearly, the threshold search cost will be higher with $u>w$ and lower with $u<w$. Furthermore, in the presence of search costs and demand uncertainty, availability guarantees serve as a useful contractual device to stimulate demand. We offer two intuitive explanations for why such guarantees are attractive measures to adopt. First, by offering a monetary compensation, the seller can charge a higher price and extract more profit. This is similar in spirit to some signaling games where firms "burn cash" or impose self-punishment to signal their quality. It has also been widely acknowledged that price and advertising are also effective signaling tools (see Milgrom and Roberts, 1986 and Dana, 2001). Here, by offering availability guarantees, the seller is inflating his own costs in the event of stockouts. Doing so indirectly convinces consumers that the service level will be high, since stockouts are costly to the seller. Indeed, observe from (12) in Proposition 4 that the seller's underage cost is increased as a result of $w$. Moreover, observe from (10) that the possibility of receiving $u$ in the event of stock-outs increases consumers' willingness to pay, raises the seller's profit margins, and create incentives for the seller to improve service levels, thus reinforcing consumer faith in the seller. Second, since consumers recognize the possibility of stockouts before visiting the seller, they will never pay full valuation for the product. Indeed, to induce demand, the seller must pay consumers a premium to cover the possibility of facing a stockout. This could take two forms: an ex-ante premium (in the form of a reduction in price) or an ex-post premium (in the form of the stockout compensation). Notice that ex-ante premia are always enjoyed by all consumers while ex-post premia applies only when stockouts occur. Thus, from the perspective of the seller, offering availability guarantees is a more cost-effective way to stimulate demand, compared to reducing the price. 


\subsection{Monetary Compensation}

We next consider the special case of monetary compensation with $w=u$. That is, the seller offers a fixed monetary compensation $u>0$ to consumers who experience a stockout. With this setup, we consider the following questions. First, how do monetary guarantees affect the equilibrium price and quantity? Second, how much compensation $u$ should the seller offer? Specifically, should the seller offer maximal compensation $u=h$ ? Third, with an optimal compensation scheme, how much does the seller make, compared to our previous scenarios? The following two propositions provide answers to all these questions. The subscript $M$ stands for monetary compensation.

Proposition 5 The seller's equilibrium price $p_{M}^{*}$, quantity $q_{M}^{*}$ and profit $\Pi_{M}^{*}$ are increasing in $u$.

Proposition 6 Suppose the seller offers maximal monetary compensation $u=h$. Then, the RE equilibrium satisfies $p_{M}^{*}=v-h>p_{C}^{*}, q_{M}^{*}=q_{C}^{*}=\bar{F}^{-1}(c / v)$, and $\Pi_{M}^{*}=\Pi_{C}^{*}$.

These results demonstrate that the seller should offer maximal monetary compensation $u=h$. There are two noteworthy implications. First, this implies that the seller should fully reimburse the search cost to consumers who face stockouts. This places all consumers in an enviable risk-free situation: regardless of whether each consumer finds the product in stock, the consumer will never incur any losses ex-post. In fact, by offering to cover search costs, the seller may now price at consumers' full valuation (net of search costs), i.e., $p=v-h$; note that this is the highest possible price that the seller may charge. Therefore, with maximal compensation $u=h$, potential consumer losses are completely eliminated but it is the seller who then extracts the entire consumer surplus. Second, the seller can attain the profit under commitment $\Pi_{C}^{*}$ through availability guarantees. Without any monetary compensation, the seller has to contend with the RE equilibrium profit $\Pi^{*}$; in contrast, with maximal monetary compensation $u=h$, the seller is able to attain profit level $\Pi_{C}^{*}$. This suggests that monetary compensation can serve as a proxy for commitment power, both of which can be used by the seller to influence consumer behavior to enhance profit.

\subsection{Efficient Guarantees}

We shall now consider more general forms of availability guarantees beyond monetary compensation with $w=u$. Typically, firms have the opportunity to exploit cost savings by offering guarantees with $w \leq u$, such that consumers who face stock-outs derive utility $u$ from the compensation but this costs the firm only $w$. This is possible, for instance, by offering free gifts or discount coupons 


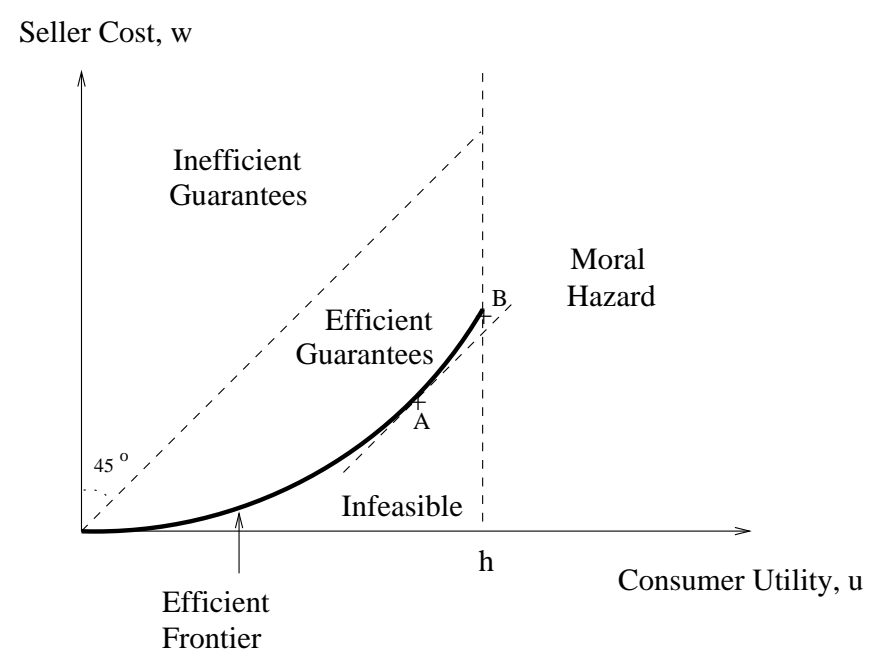

Figure 1: Different formats of availability guarantees.

since the firm's own profit margin drives a wedge between the two parameters $u$ and $w$. However, since cost savings are not limitless, to maintain realism, we assume that in order to provide stockedout consumers with utility $u$, there is a minimum cost $\underline{W}(u)$ that the firm must incur. We shall refer to this as the efficient frontier, and we assume that $\underline{W}(u)$ is increasing and convex in $u$. Figure 1 shows the feasible formats of availability guarantees (i.e., different $(u, w)$ combinations) that the firm can choose from. The bold curve represents the efficient frontier, below which are infeasible $(u, w)$ combinations; similarly, we do not allow $u>h$ in order to prevent consumer moral hazard. The dashed 45-degree line corresponds to guarantees based on monetary compensation, as analyzed previously. Feasible combinations below this line are efficient guarantee formats $(w \leq u)$ while those above are inefficient formats $(w \geq u)$.

As a first step in our analysis, we consider the following guarantee format: $u=h$ and $w=\underline{W}(h)$, i.e., point B in Figure 1. This maximal compensation fully insures consumers for their search cost $h$, and also exploits cost savings to some extent. In particular, these cost savings provide additional room for the seller to do better than the commitment-benchmark of Section 4. We have already seen that this profit benchmark $\Pi_{C}^{*}$ can be attained through the availability guarantee with monetary compensation $u=h$. The next result confirms that exploiting cost savings allows the seller to do even better.

Proposition 7 Suppose the seller offers an availability guarantee with $u=h$ and $w=\underline{W}(h)$. Then, the $R E$ equilibrium satisfies $p_{G}^{*}=v-h \geq p_{C}^{*}, q_{G}^{*} \leq q_{C}^{*}$, and $\Pi_{G}^{*} \geq \Pi_{C}^{*}$. 
This result shows that, similar to the case with maximal monetary compensation $u=h$, the equilibrium price also reaches the highest possible price of $v-h$, which exceeds the equilibrium price $p_{C}^{*}$ in the commitment-benchmark. However, different from the case with maximal monetary compensation $u=h$ is that the equilibrium quantity here is lower than $q_{C}^{*}$ under commitment. This is because the cost saving discussed above decreases the seller's underage cost and induces him to stock less. Nevertheless, with lower stocking quantity, the seller still attains higher profits, compared to both the maximal monetary compensation $u=w=h$ case and the commitmentbenchmark case.

Finally, we close this section by analyzing the seller's optimal choice of guarantee formats $(u, w)$. We introduce some notation. Let $\left(u^{* *}, w^{* *}\right)$ denote the feasible $(u, w)$ pair that maximizes the difference $u-w$. This can be interpreted as the socially efficient availability format, which generates the highest net benefit when stockouts occur. This socially efficient point is marked as point A in Figure 1.

Proposition 8 Let the seller's profit-maximizing guarantee format be $\left(u^{*}, w^{*}\right)$. Then, we have (i) $u^{*} \geq u^{* *}$ and $(i i) w^{*}=\underline{W}\left(u^{*}\right)$.

Interestingly, this proposition shows that while the profit-maximizing seller will fully exploit cost savings by choosing a guarantee format on the efficient frontier (part ii), he has an incentive to over-compensate (part i). When left to his own devices, the seller's availability guarantees are too generous because the compensation paid to consumers during stock-outs $u^{*}$ exceeds the socially efficient level $u^{* *}$. Therefore, the marginal value of the last unit of compensation provided by the seller is negative. To see this, observe that the slope of the efficient frontier at the seller's choice $u^{*}$ is $\underline{W}^{\prime}\left(u^{*}\right) \geq 1$. The consumer values this marginal unit of compensation at less than the resources required to provide it, and this leads to deadweight losses. This inefficiency can be attributed to the standard monopoly problem, wherein the monopolist sets too high a price; in our context, an excessively generous guarantee is offered in order to realize a high price.

\subsection{Inefficient Guarantees}

We provide a brief discussion about inefficient guarantees in this section. A direct consequence of our result in Proposition 8 is that inefficient guarantees are suboptimal for the seller. Although this finding is not surprising, we wish to caution readers of the possible boundaries of this result. After all, it is conceivable that offering inefficient guarantees may allow the seller to better commit 
to providing high service levels, since the cost of compensating consumers is now even higher (relative to using efficient guarantees). We conjecture that such commitment strategies, via offering inefficient guarantees, may be desirable in competitive environments. In a different context, Kim, Shi, and Srinivasan (2001) discuss efficient versus inefficient rewards for customer loyalty.

\section{First Best}

In this section, we take on a social welfare perspective. Assuming that there is a social planner who is able to influence the actions of consumers and firms (prior to demand realization), what should this social planner do? This is in contrast to the equilibrium analyses from previous sections, in which consumers and the seller were assumed to maximize individual payoffs. By comparing the outcomes of individual versus social optimization, our goal is to identify the sources of efficiency loss and to design mechanisms that achieve first-best.

We begin our analysis by defining the social welfare function. Recall that the seller sets the price $p$ and quantity $q$, while the consumers select reservation prices $r$. In addition, the seller may choose the type of availability guarantee $(u, w)$ to provide. Since prices merely constitute transfer payments and consumers need to visit the seller in order to realize non-zero social surplus, we may disregard $p$ and $r$ and focus on the social planner's choices of $q$ and $(u, w)$. Accordingly, the social welfare function may be expressed as

$$
S W(q, u, w)=v E(X \wedge q)-c q-h E(X)+(u-w)[E(X)-E(X \wedge q)]
$$

Each of the four terms respectively correspond to: (i) expected valuation enjoyed by the consumer population, (ii) production cost, (iii) search cost incurred by all consumers, and (iv) expected social surplus generated from compensating consumers facing stockouts. With this characterization, it is straightforward to find the first-best quantity $q^{F B}$ and guarantee format $\left(u^{F B}, w^{F B}\right)$ that maximize social welfare.

Proposition 9 The first-best quantity and guarantee format are given by

$$
\begin{aligned}
\bar{F}\left(q^{F B}\right) & =\frac{c}{v-\left(u^{* *}-w^{* *}\right)} \\
\left(u^{F B}, w^{F B}\right) & =\left(u^{* *}, w^{* *}\right) .
\end{aligned}
$$

In general, the first-best compensation utility is below the maximal level, i.e., $u^{F B}=u^{* *} \leq h$,

and the first-best quantity satisfies $q^{F B} \leq \bar{F}^{-1}(c / v)$. To see the significance of these comparisons, 
let us consider two hypothetical scenarios, one where the seller bears the entire burden of potential stockouts, and another where consumers bear the entire burden. The former occurs when the seller offers maximal compensation $u=h$, which ensures that consumers never suffer negative ex-post payoffs. The latter corresponds to the situation in which all consumers unconditionally visit the seller and are willing to pay their full valuation $v$, which then leads the seller to stock quantity $q=\bar{F}^{-1}(c / v)$. Observe that the first-best outcome falls between these two extremes. This suggests that social optimality requires the seller and the consumers to jointly share the potential downside of supply-demand mismatches.

Unsurprisingly, we find that the first-best outcome is not attained in any of the scenarios considered above. In the absence of availability guarantees (i.e., when $u=0$ ), the first-best is not achieved. This applies to our basic model, as well as our commitment-benchmark. However, even when availability guarantees are considered, the RE equilibrium still does not sustain firstbest outcomes. When we focus on monetary compensation, the seller's profit-maximizing choice of $u$ is $u=h$. Although this attains the commitment-benchmark outcomes, the first-best is not achieved because potential cost savings from alternative types of availability guarantees have not been made use of. When we consider efficient guarantees, recall our earlier finding that the seller's profit-maximizing compensation level exceeds the socially efficient level, i.e., $u^{*} \geq u^{F B}$. Again, the first-best is not attained in equilibrium.

Fortunately, as we illustrate below, the first-best can be attained in RE equilibrium by using a combination of commitment and availability guarantees. To see this, consider the situation in which the seller can commit to a stocking quantity $q$ and on top of this, the seller provides availability guarantee of type $(u, w)$. In this case, given the seller's quantity $q$, consumers form their reservation prices $r$ via the expression $(v-r) A(q)+u(1-A(q))=h$. Therefore, corresponding to quantity $q$, the seller charges the price $p(q)$ satisfying

$$
(v-p(q)) A(q)+u \bar{A}(q)=h .
$$

It then follows that the seller's profit function is

$$
\begin{aligned}
\Pi_{B}(q, u, w) & =p(q) E(X \wedge q)-c q-w[E(X)-E(X \wedge q)] \\
& =\left(v-u-\frac{h-u}{A(q)}\right) E(X \wedge q)-c q-w[E X-E(X \wedge q)] \\
& =v E(X \wedge q)-c q-h E(X)+(u-w)[E X-E(X \wedge q)] \\
& =S W(q, u, w),
\end{aligned}
$$


which coincides with the social welfare function. In this way, the seller's individual interests are aligned with social welfare, so he chooses $q_{B}^{*}=q^{F B}$ and $\left(u_{B}^{*}, w_{B}^{*}\right)=\left(u^{F B}, w^{F B}\right)$, thus attaining social welfare. Here, the subscript B refers to "both commitment and availability guarantees". The following proposition summarizes these findings.

Proposition 10 Suppose that the seller can commit to quantity and implements a profit-maximizing availability guarantee format. Then, in RE equilibrium, the first-best is attained, with $q_{B}^{*}=q^{F B}$ and $\left(u_{B}^{*}, w_{B}^{*}\right)=\left(u^{F B}, w^{F B}\right)$. Moreover, the equilibrium price is

$$
p_{B}^{*}=\left(v-u^{F B}-\frac{h-u^{F B}}{A\left(q^{F B}\right)}\right) \leq v-h .
$$

Finally, the seller extracts the entire social surplus and consumers' expected surplus is zero.

The main thrust of this result is to confirm that the seller can indeed implement the first-best in a RE equilibrium. Doing so requires a combination of two things: a commitment device for the seller to provide credible inventory information prior to demand realization, as well as an incentive mechanism to induce demand by compensating consumers for potentially adverse outcomes afterwards. Both time points (i.e., before and after) must be taken care of. In isolation, each of the strategies proposed above (i.e., establishing commitment power and providing availability guarantees) is not sufficiently powerful to realize the first-best, but when appropriately combined, the inherent synergies can allow the seller to capture the entire first-best surplus.

\section{Extensions}

\subsection{Learning}

This section studies a multi-period extension with consumer learning. In the static model of Section 3 , the RE equilibrium outcomes are natural and intuitive, provided that players possess rational expectations. In other words, our basic model applies in the long run when equilibrium beliefs have formed and stabilized. Now, we also consider the transient setting. We outline a dynamic model of consumer learning and investigate whether market outcomes and beliefs indeed converge toward the static RE equilibrium analyzed earlier.

In this model extension, the seller interacts with a fixed market of consumers over discrete time periods $t=1,2, \ldots$ In each period $t$, consumers have some belief $\hat{\xi}_{t}$ over the availability probability. Similar to the basic model, these beliefs determine consumers' reservation price, so 
the seller can charge up to price $p_{t}=v-h / \hat{\xi}_{t}$. The seller also chooses a stocking quantity $q_{t}$, so the actual availability probability in period $t$ will be $\xi_{t}=A\left(q_{t}\right)$. At the end of each period, the consumer population updates the belief for the next period to be $\hat{\xi}_{t+1}=\alpha \xi_{t}+(1-\alpha) \hat{\xi}_{t}$ for some $\alpha \in(0,1)$. Such an exponential learning method have been widely used in the literature (see Liu and van Ryzin, 2007 and the references therein). While each period in this dynamic model can be viewed as a separate replica of our basic model, consumers' beliefs in one period do influence their beliefs in the next period.

Now, the seller faces a dynamic optimization problem instead of a static newsvendor problem. Let $\Pi(q, \hat{\xi})$ denote the seller's profit from stocking $q$ units in a period when consumer beliefs are $\hat{\xi}$. Since the seller charges price $p=v-h / \hat{\xi}$, the expected profit in this period is

$$
\Pi(q, \hat{\xi})=(v-h / \hat{\xi}) E(X \wedge q)-c q
$$

The seller wishes to maximize total discounted profit over an infinite horizon $\sum_{t=1}^{\infty} \delta^{t-1} \Pi\left(q_{t}, \hat{\xi}_{t}\right)$. Therefore, we can write down the Bellman equations for the seller's dynamic program as follows:

$$
V(\hat{\xi})=\max _{q}[\Pi(q, \hat{\xi})+\delta V(\alpha A(q)+(1-\alpha) \hat{\xi})] .
$$

Then, the maximizer $q^{*}(\hat{\xi})$ in the equation above yields the seller's optimal quantity decision when consumer beliefs are $\hat{\xi}$. Furthermore, we may interpret the value function $V(\hat{\xi})$ as the seller's optimal total discounted profit, given current beliefs $\hat{\xi}$.

Under the seller's optimal policy, his quantity decisions $q_{t}^{*}$ and consumers' beliefs $\hat{\xi}_{t}$ in each period $t$ are governed by the following dynamic system:

$$
\begin{array}{ll}
q_{1}^{*}=q^{*}\left(\hat{\xi}_{1}\right), & \hat{\xi}_{2}=\alpha A\left(q_{1}^{*}\right)+(1-\alpha) \hat{\xi}_{1}, \\
q_{2}^{*}=q^{*}\left(\hat{\xi}_{2}\right), & \hat{\xi}_{3}=\alpha A\left(q_{2}^{*}\right)+(1-\alpha) \hat{\xi}_{2}, \ldots \\
q_{t}^{*}=q^{*}\left(\hat{\xi}_{t}\right), \quad \hat{\xi}_{t+1}=\alpha A\left(q_{t}^{*}\right)+(1-\alpha) \hat{\xi}_{t}, \ldots
\end{array}
$$

We are interested in the long run behavior of this dynamic system. Do the seller's optimal quantities $q_{t}^{*}$ and consumers' beliefs $\hat{\xi}_{t}$ converge to some steady state? The next proposition shows that the answer is yes and characterizes the long run equilibrium quantity and belief.

Proposition 11 Let the initial belief be $\hat{\xi}_{1}$. There exists some threshold $z \in[0,1]$ such that:

(i) if $\hat{\xi}_{1}<z$, then $q_{t}^{*} \rightarrow 0$ and $\hat{\xi}_{t} \rightarrow 0$.

(ii) if $\hat{\xi}_{1}>z$, then $q_{t}^{*} \rightarrow \widetilde{q}$ and $\hat{\xi}_{t} \rightarrow \widetilde{\xi}$, where $\widetilde{\xi}=A(\widetilde{q}) \in(0,1)$ and $\widetilde{q}>0$ is the larger solution of

$$
\left(v-\left(\frac{1-\delta}{1-(1-\alpha) \delta}\right) \frac{h}{A(q)}\right) \bar{F}(q)-c=0 .
$$


In particular, under Assumption 1 (i.e., $h<\bar{h}$ ), we have $z<1$.

This proposition shows that in our model of adaptive learning, there are two possible long run equilibria. In case (i) of the proposition, quantities and beliefs converge toward zero, so the market eventually breaks down in the long run. In case (ii), however, there is some non-zero equilibrium that is eventually attained and then persists over time. Let us call this equilibrium (i.e., quantity $\widetilde{q}$, consumer beliefs $\widetilde{\xi}=A(\widetilde{q})$, and price $\widetilde{p}=v-h / \widetilde{\xi})$ the long run equilibrium. The proposition above also shows that this long run equilibrium will emerge as long as consumer search $\operatorname{cost} h$ is not too large and consumers are sufficiently optimistic at the start (i.e., $\hat{\xi}_{1}$ is sufficiently large).

Interestingly, the long run equilibrium coincides with a static RE equilibrium in our basic model. The next result draws this connection.

Proposition 12 Let $q^{*}[h]$ be the static RE equilibrium quantity when consumers face search cost h. Then, the long run equilibrium quantity $\widetilde{q}$ satisfies

$$
\widetilde{q}=q^{*}\left[\frac{1-\delta}{1-(1-\alpha) \delta} \cdot h\right]
$$

This proposition shows that the long run equilibrium in our dynamic model is precisely the RE equilibrium for our static model, with some appropriately adjusted consumer search cost. When the discount factor is $\delta$, the long run quantity $\widetilde{q}$ matches the static RE quantity $q^{*}$ with an adjusted search cost of $\frac{1-\delta}{1-(1-\alpha) \delta} \cdot h \leq h$. This downward adjustment suggests that in the dynamic equilibrium, consumers are equivalently playing the static RE equilibrium but with a smaller search cost. The intuition is as follows. In a dynamic setting, the seller's quantity decision in any given period influences future beliefs and future profits, so there is a greater incentive to maintain high service levels. Such reputation effects decrease stockout experiences, so consumers can behave as if their search costs were lowered. From a modeling perspective, this result provides a tight link between the static and dynamic models. This link demonstrates that players can indeed reach a static RE equilibrium through a simple adaptive learning process.

Finally, notice that as the discount factor $\delta \rightarrow 1$, the long run equilibrium approaches the commitment benchmark. To see this, note that $\frac{1-\delta}{1-(1-\alpha) \delta} \rightarrow 0$ as $\delta \rightarrow 1$, so from equation (25) of Proposition 11, the long run quantity $\widetilde{q} \rightarrow \bar{F}^{-1}(c / v)$, which is the seller's optimal quantity with commitment (cf. Proposition 2). This comparison suggests that in a dynamic setting with consumer learning, the seller's reputation concerns (when $\delta \approx 1$ ) can serve as a surrogate for commitment power. 


\subsection{A multi-product model}

This section demonstrates that the basic model can be extended to incorporate multiple products. Suppose the seller offers consumers a set of products indexed by $i=1, \ldots, n$. We assume that the set of products is fixed. For instance, a retailer sells shirts from a fixed set of different sizes. There is a pool of consumers and each consumer demands one unit of the product. However, a consumer does not know which product is suitable before she visits the seller, e.g., a consumer needs to try the shirt on in order to know the right size. Assume that there is at most one suitable product for each consumer. In other words, a lost sale occurs if a consumer's first choice is unavailable. This model has been used in van Ryzin and Mahajan (1999) and is a special case of the models studied by Smith and Agrawal (2000) and Mahajan and van Ryzin (2001). The consumers are divided into $M$ types according to certain characteristics (e.g., height and weight). The fraction of type $j(1 \leq j \leq M)$ consumers is denoted by $\alpha_{j}\left(\sum_{j} \alpha_{j}=1\right)$ and let $\lambda_{i j}$ denote the probability that product $i$ is suitable for a type- $j$ consumer $\left(\sum_{i} \lambda_{i j}=1\right.$ for all $\left.j\right)$. The products share the same unit cost $c$. For ease of exposition, we assume that all consumers have an identical valuation $v$ for a suitable product and zero otherwise.

First consider the consumer's problem. If a type- $j$ consumer chooses to visit the seller, she will get an expected surplus $\sum_{i} \lambda_{i j}\left(v-p_{i}\right) \xi_{i}-h$, where $\xi_{i}$ is the availability probability and $p_{i}$ is the price for product $i$. She receives zero surplus if she does not visit the seller.

Next consider the seller's problem. Assume all consumers choose to visit the seller. Then the demand for product $i$ is $\left(\sum_{j} \alpha_{j} \lambda_{i j}\right) X$. Define $\lambda_{i}=\sum_{j} \alpha_{j} \lambda_{i j}$ as the fraction of demand for product $i$. Since $\sum_{j} \alpha_{j}=1$ and $\sum_{i} \lambda_{i j}=1$, we know $\sum_{i} \lambda_{i}=1$. Given the price $p_{i}$, the optimal stocking quantity $Q_{i}$ is given by $\operatorname{Pr}\left(\lambda_{i} X \leq Q_{i}\right)=\left(p_{i}-c\right) / p_{i}$. Given $Q_{i}$, the availability probability is then $\xi_{i}=\frac{E\left(\lambda_{i} X, Q_{i}\right)}{E\left(\lambda_{i} X\right)}=\frac{E\left(X, Q_{i} / \lambda_{i}\right)}{E(X)}=A\left(Q_{i} / \lambda_{i}\right)$, which only depends on $p_{i}$. For convenience, define $\hat{Q}_{i}=Q_{i} / \lambda_{i}$ as the effective quantity. Thus, once $p_{i}$ is chosen, both the seller's optimal effective quantity $\hat{Q}_{i}$ (in equilibrium) and the availability probability $\xi_{i}=A\left(\hat{Q}_{i}\right)$ are determined.

Recall in the basic model with a single product, the price $p$ is determined in the RE equilibrium. In particular, the seller would charge a price such that the consumer's expected surplus is zero, i.e., $(v-p) \xi-h=0$. Similarly, with multiple products, the seller will choose a price such that consumer surplus $\sum_{i} \lambda_{i j}(v-p) \xi_{i}-h=0$ for all consumer types $j$. We assume that the seller charges a uniform price $p$ across all product variants (e.g., the price is the same for shirts in different sizes or yogurt with different flavors). Although the seller in theory can price discriminate, in practice, such price 
differentials are often perceived as unfair and will attract consumer resentment (see Anderson and Simester, 2005). We have the following equilibrium result.

Proposition 13 Suppose the seller charges a uniform price for all products. Then the seller will choose the same $\hat{Q}$ for all products and the $R E$ equilibrium outcome $(p, \hat{Q})$ is characterized by

$$
\begin{aligned}
\bar{F}(\hat{Q}) & =c / p \\
(v-p) \xi(\hat{Q}(p))-h & =0 .
\end{aligned}
$$

This proposition indicates that the multi-product model can be reduced to the single-product model studied in the previous sections. Therefore, the results obtained for the basic model also apply to the multi-product setting.

\subsection{Heterogeneous consumer valuations}

This section extends the basic model in Section 3 to consider heterogeneous consumers. Now consumer valuation is continuously distributed on a support $[0, v]$. We index consumers by $\theta$ such that consumers' valuation $v_{\theta}$ increases in $\theta$. Let $\Phi\left(v_{\theta}\right)$ be the proportion of consumers with a valuation $v_{\theta}$ or lower. (Note that the homogeneous model is a special case with a point distribution at $v$.) The total market demand is still $X$ with a distribution function $F$, which is independent of the distribution $\Phi$. Therefore, if the consumer segment that has a valuation $v_{\theta}$ or higher chooses to visit the seller, the seller's random demand will be $\left(1-\Phi\left(v_{\theta}\right)\right) X$. As before, there is a search cost $h$ for all consumers. The seller sets an observable price $p$ and an unobservable quantity $q$ before the random demand $X$ is realized. The consumers decide whether to visit the seller or not.

Suppose the consumers observe the seller's price and form a belief of the availability probability. Let $\theta$ be the marginal consumer who is indifferent between visiting and not visiting the seller. Define $s\left(v_{\theta}\right)=1-\Phi\left(v_{\theta}\right)$ as the market base for the seller (i.e., the fraction of the market that will choose to visit the seller). We assume proportional rationing for this heterogeneous model: all consumers who choose to visit the seller have equal chances of getting the product. ${ }^{2}$ Under proportional

\footnotetext{
${ }^{2}$ Another plausible rationing scheme is efficient (or parallel) rationing, which assumes that products are assigned to consumers in decreasing order of their valuations. A possible justification is that higher-valuation consumers are more eager and hence more likely to secure the product in the event of a shortage. Under efficient rationing, the marginal consumer (lowest-valuation consumer who chooses to visit the seller) has the lowest priority. Therefore, ceteris paribus, efficient rationing will lead to a smaller market base for the seller. In a capacity pricing model, Dana (1998) considers both proportional and efficient rationing schemes and obtains similar results for both cases.
} 
rationing, in any $\mathrm{RE}$ equilibrium, the belief of availability probability $\xi_{\text {prob }}$ must agree with the actual outcome corresponding to the seller's quantity $q$ and the marginal customer's valuation $v_{\theta}$ :

$$
\xi_{\text {prob }}=\frac{E\left(s\left(v_{\theta}\right) X \wedge q\right)}{E\left(s\left(v_{\theta}\right) X\right)}=\frac{E\left[X \wedge\left(q / s\left(v_{\theta}\right)\right)\right]}{E(X)}=A(z),
$$

where $z=q / s\left(v_{\theta}\right)$ and $A(z)=\frac{E[X \wedge z]}{E(X)}$.

Suppose the seller offers an availability guarantee with utility-cost pair $(u, w)$. For the same reasons in Section 5, we focus on $0 \leq u \leq h$. Below we derive and compare the RE equilibria under two scenarios, i.e., the scenarios with and without commitment. The following proposition characterizes the conditions for the existence of RE equilibrium without commitment. The subscript $H$ refers to heterogeneous consumers.

Proposition 14 Consider the heterogeneous RE game without quantity commitment. Under availability guarantee $(u, w)$ :

(i) There exists $\bar{h}_{H} \in(0, h]$ such that a $R E$ equilibrium exists only if $h-u<\bar{h}_{H}$.

(ii) For $h-u<\bar{h}_{H}$, let $z_{1}<z_{2}$ be the two solutions to $v-\frac{c}{\bar{F}(z)}+\frac{u-h}{A(z)}=0$ and $z_{3}$ be the unique solution to $\frac{c}{\bar{F}(z)} E(X \wedge z)-c z-w E(X)=0$. Then a RE equilibrium exists if and only if $z_{2}>z_{3}$. In any equilibrium, the seller's price $p$, quantity $q$, and the marginal valuation $v_{\theta}$ (and therefore the market base $\left.s\left(v_{\theta}\right)\right)$ satisfy

$$
\begin{aligned}
\left(v_{\theta}-p\right) A\left(q / s\left(v_{\theta}\right)\right)+u\left(1-A\left(q / s\left(v_{\theta}\right)\right)\right) & =h, \\
\bar{F}\left(q / s\left(v_{\theta}\right)\right) & =c /(p+w) .
\end{aligned}
$$

The above proposition states that a RE equilibrium does not exist in the heterogeneous model when the search cost $h$ is too high. Similar to the homogeneous model, a stockout compensation $u>0$ might be needed in order to prevent market failure. The proposition also indicates that multiple RE equilibria may exist in the heterogeneous model. In fact, for any particular $z\left(z_{3}<\right.$ $\left.z<z_{2}\right)$, there exists a corresponding $\mathrm{RE}$ equilibrium $\left(p, z, s\left(v_{\theta}\right)\right)$ that satisfies (27) and (28) and yields a positive profit for the seller. This means that in the heterogeneous model, the seller first determines the optimal value for $z$ (or the market base $s\left(v_{\theta}\right)$ ), and then plays the corresponding RE game with the consumers.

Next we consider the scenario with commitment. In this scenario, the seller can freely choose both price $p$ and quantity $q$. The market base $s\left(v_{\theta}\right)$ is then given by the consumers' indifference condition, i.e. $\left(v_{\theta}-p\right) A\left(q / s\left(v_{\theta}\right)\right)+u \bar{A}\left(q / s\left(v_{\theta}\right)\right)=h$. This is in contrast with the no-commitment 
scenario above where the seller can only choose the market base and the rest of the decisions are governed by the equilibrium conditions. Note there is a one-to-one relationship between $q$ and $z=q / s\left(v_{\theta}\right)$. Thus solving the RE equilibrium is equivalent to identifying the optimal $p$ and $z$ that maximize the seller's profit.

With commitment power, the seller can replicate any RE equilibrium that may arise without commitment. This implies that the seller's profit in the commitment scenario is always higher than that in the no-commitment scenario. Further, plugging $u=h$ into (27) gives $v_{\theta}-p=h$, which does not contain $z$. In this case, the $z$ decision is decoupled from the $p$ decision in the no-commitment scenario. As a result, the seller's optimal decisions (including the corresponding profit) in the nocommitment scenario will be the same as those in the commitment scenario. Therefore, we know that the seller's profit in the no-commitment scenario converges to the profit in the commitment scenario from below as $u$ approaches $h$ (see Figure 2 for an example).

What is the impact of availability guarantees on the seller's profit and social welfare? Do the results from the homogeneous model continue to hold in the heterogeneous model? The rest of this section aims to answer these questions. Note that with heterogeneous consumer valuations, it is infeasible for the seller to extract the entire social surplus by charging a single price. Nevertheless, we may still examine whether the seller's individual interests are aligned with social welfare. The following proposition demonstrates that the seller will choose the socially efficient guarantee with commitment.

Proposition 15 Consider the heterogeneous RE game with quantity commitment. Let the seller's profit-maximizing guarantee format be $\left(u^{*}, w^{*}\right)$. Then, $u^{*}=u^{* *}$ and $w^{*}=\underline{W}\left(u^{*}\right)$.

Characterizing the seller's optimal guarantee in the no-commitment scenario is analytically challenging, so we use extensive numerical experiments to derive additional insight. In particular, we are interested in how the seller's profit and social welfare depend on the compensation level $u$ in both scenarios. For sake of brevity, we present the results from a representative numerical example. In this example, $\Phi$ is uniform on [0,1] (i.e., $v=1$, without losing generality) and other parameter values are as follows: $c=0.4, h=0.2$, the cost of availability guarantee is $\underline{W}(u)=10 u^{2}$, and $X$ follows a gamma distribution with mean 90 and standard deviation 30 . The socially efficient compensation level is $u^{* *}=0.05$. Figure 2 shows how the seller's profit and the corresponding social welfare vary in the compensation level level, $u$. We can see from Figure 2 that both the seller's profit and the social welfare are higher with quantity commitment than their counterparts without 


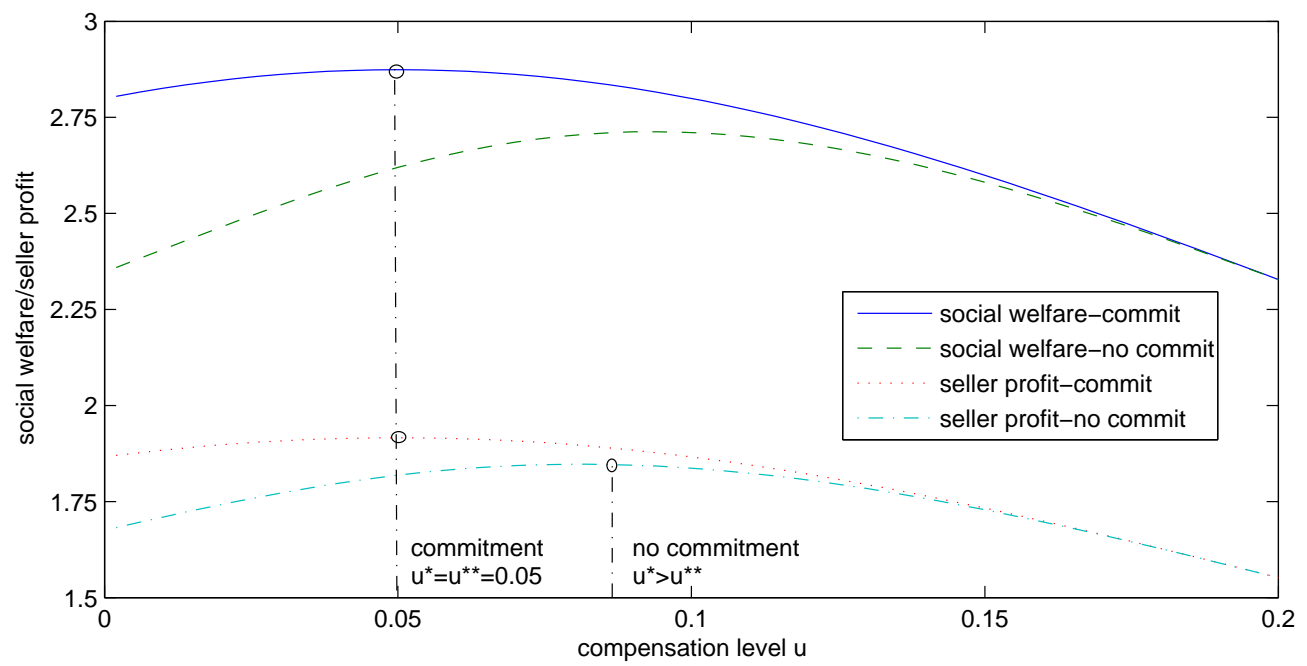

Figure 2: Seller profit and social welfare functions.

commitment. In addition, there is $u^{*}=u^{* *}=0.05$ with commitment, i.e., the seller's profitmaximizing compensation level is socially efficient, which also delivers the highest level of social welfare. However, the seller will over-compensate (i.e., $u^{*}>u^{* *}$ ) in the no-commitment scenario. From the numerical study, we observe that quantity commitment and availability guarantees can improve social welfare as well as the seller's profit in the model with consumer heterogeneity. Also, both quantity commitment and availability guarantees are required in order to realign the seller's individual interests with social welfare.

Notice that an alternative way to model consumer heterogeneity is by introducing heterogeneous search costs. One practical example is when consumers are dispersed geographically so they incur different transportation costs in visiting the seller. The analysis for heterogeneous search costs is quite different from that for heterogeneous valuations. Recall that with heterogeneous valuations, the marginal consumer is determined by the indifference equation $\left(v_{\theta}-p\right) A\left(q / s\left(v_{\theta}\right)\right)=h$ (assuming stockout compensation $u=0$ ), where $v_{\theta}$ is the marginal valuation. With heterogeneous search costs, the analogous indifference equation becomes $(v-p) A\left(q / s\left(h_{y}\right)\right)=h_{y}$, where $h_{y}$ is the search cost for the marginal consumer type $y$ and $s\left(h_{y}\right)$ is the market base given $h_{y}$. Thus, the two modeling approaches generate indifference conditions with different mathematical structures. How the above results will change when consumers differ in their search costs is an open question that should be addressed in future research. 


\section{Conclusion}

In this paper, we study the impact of product availability on market outcomes. High service levels induce consumer demand by lowering the chances of stockout, but must be correspondingly supported by the firm's inventory investment. We develop a modeling framework based on the rational expectations paradigm, and characterize the seller's equilibrium price and inventory levels when facing rational consumers who alter their shopping behavior in response to the anticipated likelihood of stockouts. We study two strategies that can be used to attract consumer demand. The first strategy is to use an external commitment device, i.e., the seller credibly promises to keep service levels high; the second is to offer availability guarantees, i.e., the seller promises to compensate consumers in the event of a stockout. Our main conclusion is: the seller may enhance profits by either using commitment power or providing availability guarantees, and may additionally realize first-best outcomes via a combination of both strategies.

This research can be extended in several directions. First, we have considered only a single product, whereas in practice, most firms offer multiple substitutable products, which offer opportunities for substitution when stockouts occur. For example, consumers at a video rental store may switch to their second-choice movie when their first-choice is not available. Similarly, fashion apparel that come in different designs and colors offer additional options when stockouts occur. This suggests that with ex-post consumer substitution, product variety can serve as a strategic lever for firms to alleviate concerns about product availability. A complete investigation is left for future research. Second, consumer heterogeneity is present in most markets. Although we have briefly studied the case where consumers have different valuations, it would also be interesting to consider the case where consumers have different search costs. The interaction (correlation) between these two dimensions of consumer heterogeneity may also have strategic implications on firms' operational policies. Finally, our analysis has assumed that consumers are "ultra-rational" in that they form rational expectations and make optimal decisions with perfect accuracy. How should our model be extended to capture mis-estimation and decision errors, which invariably do occur? We believe that incorporating consumer bounded rationality is a fruitful area for future research.

\section{References}

Alexandrov, A., M.A. Lariviere. 2007. Are reservations recommended? Working paper. 
Andersen Consulting. 1996. Where to look for incremental sales gains: The retail problem of out-of stock merchandise. The Coca-Cola Retailing Research Council, Atlanta.

Anderson, E.T., G.J. Fitzsimons, D. Simester. 2006. Measuring and mitigating the costs of stockouts. Mgmt. Sci. 52(11): 1751-1763.

Anderson, E.T., D. Simester. 2005. Does demand fall when customers perceive that prices are unfair: The case of premium pricing for large sizes. Working paper.

Aviv, Y., A. Pazgal. 2008. Optimal pricing of seasonal products in the presence of forward-looking consumers. MSOM 10(3): 339-359.

Balachander, S., P.H., Farquhar. 1994. Gaining more by stocking less: a competitive analysis of product availability. Mktg. Sci. 13(1): 3-22.

Besanko, D., W.L. Winston. 1990. Optimal price skimming by a monopolist facing rational consumers. Mgmt. Sci. 36(5): 555-567.

Bhargava, H.K., D. Sun, S.H. Xu. 2006. Stockout compensation: joint inventory and price optimization in electronic retailing. Journal of Computing 18(2): 255-266.

Breugelmans, E., K. Campo, E. Gijsbrechts. 2006. Opportunities for active stock-out management in online stores: The impact of the stock-out policy on online stock-out reactions. J. Retailing. 82(3): 215-228.

Bulow, J.I. 1982. Durable-goods monopolists. J. Polit. Econ. 90(2): 314-332.

Cachon, G.P., R. Swinney. 2007. Purchasing, pricing and quick response in the presence of strategic consumers. Working paper.

Coase, R.H. 1972. Durability and monopoly. J. Law \& Econ. 15(1): 143-149.

Consumer Reports. 1987. Mail-order companies. 52: 607-614.

Dana, J.D. 1998. Advance-purchase discounts and price discrimination in competitive markets. J. Pol. Econ. 106(2): 395-422.

Dana, J.D. 2000. Blockbuster video. Kellogg School of Management, Northwestern University.

Dana, J.D. 2001. Competition in price and availability when availability is unobservable. RAND J. Econ. 32(3): 497-513. 
Dana, J.D., N.C. Petruzzi. 2001. The newsvendor model with endogenous demand. Mgmt. Sci. 47(11): 1488-1497.

Dana, J.D., K.E. Spier. 2001. Revenue Sharing and Vertical Control in the Video Rental Industry. J. Industrial Econ. 49(3): 223-245.

Deneckere, R., J. Peck. 1995. Competition over price and service rate when demand is stochastic: a strategic analysis. RAND J. Econ. 26(1): 148-162.

Dong, L., N. Rudi. 2004. Who benefits from transshipment? Exogenous vs. endogenous wholesale prices. Mgmt. Sci. 50(5): 645-657.

Fitzsimons, G.J. 2000. Consumer response to stockouts. Jour. Consumer Res. 27: 249-266.

Furman, P. 1998. At Blockbuster Video, A fast fix moves flicks. Daily News. July 27.

Gruen, T.W., D. Corsten, S. Bharadwaj. 2002. Retail out of stocks: A worldwide examination of causes, rates, and consumer responses. Grocery Manufacturers of America, Washington, DC.

Gruen, T.W., D. Corsten. 2008. A comprehensive guide to retail out-of-stock reduction in the fast-moving consumer goods industry. Grocery Manufacturers of America, Washington, DC.

Hann, I.-H., C. Terwiesch. 2003. Measuring the frictional costs of online transactions: The case of a reverse buying site. Management Science 49(11): 1563-1579.

Hassin, R., M. Haviv. 2003. To Queue or Not to Queue: Equilibrium Behavior in Queueing Systems. Kluwer Academic Publishers.

Jerath, K., S. Netessine, S.K. Veeraraghavan. 2007. Revenue management with strategic customers: last-minute selling and opaque selling. Working paper.

Kim, B-D., M. Shi, K. Srinivasan. 2001. Reward programs and tacit collusion. Mktg. Sci. 20(2): 99-120.

Lai, G., L.G. Debo, K. Sycara. 2007. Impact of price matching policy on pricing, inventory investment and profit with strategic consumers. Working paper.

Liu, Q., G. van Ryzin. 2008. Strategic capacity rationing induce early purchases. Management Science 54(6): 1115-1131. 
Liu, Q., G. van Ryzin. 2007. Strategic capacity rationing when customers learn. Working paper.

Mahajan, S., G. van Ryzin. 2001. Stocking retail assortments under dynamic consumer substitution. Operations Research 49(3) 334-351.

Milgrom, P, J. Roberts. 1986. Price and advertising signals of product quality. The Journal of Political Economy 94(4): 796-821.

Muth, J.F. 1961. Rational expectations and the theory of price movements. Econometrica 29: 315-335.

Stokey, N.L. 1979. Intertemporal price discrimination. Quar. J. Econ. 93(3): 355-371.

Stokey, N.L. 1981. Rational expectations and durable goods pricing. Bell J. Econ. 12(1):112-128.

Shen, Z.J., X. Su. 2007. Customer behavior modeling in revenue management and auctions: a review and new research opportunities. POMS. 16(6): 713-728.

Smith, S.A., N. Agrawal. 2000. Management of multi-item retail inventory systems with demand substitution. Operations Research 48(1) 50-64.

Su, X. 2007. Intertemporal pricing with strategic customer behavior. Mgmt. Sci. 53(5): 726-741.

Su, X., F. Zhang. 2008. Strategic customer behavior, commitment, and supply chain performance. Management Science, forthcoming.

Sun, D., J.K. Ryan, H. Shin. 2008. Why do we observe stockless operations on the internet? Stockless operations under competition. POMS. 17(2): 139-149.

van Ryzin, G., S. Mahajan. 1999. On the relationship between inventory costs and variety benefits in retail assortments. Management Science 48(1) 50-64.

Verhoef, P.C. and L.M. Sloot. 2005. Out-of stock: Reactions, antecedents, management solutions, and a future perspective. Retailing in the 21st Century: Current and Future Trends. (eds. M. Krafft and M. Mantrala). Springer Verlag.

Video Software Dealers Association. 1999. Annual report on the home video market.

Yin, R., Y. Aviv, A. Pazgal, C.S. Tang. 2007. The implications of customer purchasing behavior and in-store display formats. Working paper. 


\section{Online Appendix: Proofs}

Proof of Proposition 1 From the equilibrium conditions of Definition 1, we see that the equilibrium price $p$ and quantity $q$ must satisfy the following equations

$$
\begin{aligned}
\bar{F}(q) & =\frac{c}{p}, \\
(v-p) A(q) & =h,
\end{aligned}
$$

which in turn give

$$
\left(v-\frac{c}{\bar{F}(q)}\right) A(q)=h
$$

We first check if there exists a solution to the above equation. Define $J(q)=\left(v-\frac{c}{\bar{F}(q)}\right) A(q)$. Notice that $J(q)<v-c$ for all $q$. Then taking derivatives yields

$$
J^{\prime}(q)=-\frac{c f(q)}{\bar{F}^{2}(q)} A(q)+(v \bar{F}(q)-c) \frac{1}{E(X)}
$$

Since $F$ has an increasing failure rate, we know that $J^{\prime}(q)$ is decreasing in $q$ and thus $J(q)$ is concave in $q$. For any RE equilibrium to exist, we need $J(q) \geq h$ at least for some $q$. Let $\hat{q}$ be the solution to $J^{\prime}(q)=0$, then the maximum value of $J(q)$ is achieved at $\hat{q}$. Clearly, a necessary condition for an equilibrium to exist is $h \leq J(\hat{q})<v-c$.

Next we check if the seller earns a positive profit in equilibrium. From (29) and (30), the seller's profit can be written as

$$
\begin{aligned}
\Pi^{*} & =\Pi\left(p^{*}, q^{*}\right)=p^{*} E\left(X \wedge q^{*}\right)-c q^{*} \\
& =\left(v-\frac{h}{A\left(q^{*}\right)}\right) E\left(X \wedge q^{*}\right)-c q^{*} \\
& =v E\left(X \wedge q^{*}\right)-c q^{*}-h E(X),
\end{aligned}
$$

where $q^{*}$ must satisfy (31). Note that for any $h<J(\hat{q})$, (31) has two solutions, $q_{1}<q_{2}$. Define $\tilde{q}=\arg \max _{q} \Pi(q)=v E(X \wedge q)-c q-h E(X)$ (i.e., $\left.\bar{F}(\tilde{q})=\frac{c}{v}\right)$, then we know $q_{2}<\tilde{q}$ by (29). This implies that the seller's profit is increasing in the equilibrium quantity $q^{*}$, which is bounded by $\tilde{q}$. Thus the seller would choose $q^{*}=q_{2}$ in the RE equilibrium. Since $q_{2}$ is the larger root of $J(q)=h$ and $J(q)$ is concave, we know that $q_{2}$ decreases in $h$, or the seller's equilibrium profit decreases in $h$. Therefore, there exists a $h^{\prime}$ such that the seller earns a positive profit if and only if $h<h^{\prime}$. Finally, define $\bar{h}=J(\hat{q}) \wedge h^{\prime}$ and notice that both $J(\hat{q})$ and $h^{\prime}$ increase in $v$ and decrease in $c$. 
Proof of Proposition 2 Observe that the seller's profit function $\Pi_{C}(q)$ is a newsvendor profit function in terms of $q$ only, so we have (7). We have already established (8) in our discussion and the expression for $\Pi_{C}^{*}$ follows immediately from substituting $q_{C}^{*}$ into $\Pi_{C}(q)$. Finally, we check if the seller makes a positive profit (i.e., $\Pi_{C}^{*}>0$ ). Recall that $h^{\prime}$ is the threshold for the seller to make a positive profit in the RE equilibrium without commitment (see the proof of Proposition 1). Define $\bar{h}_{C}$ as the threshold such that $\Pi_{C}^{*}=0$ (note that $\Pi_{C}^{*}$ is decreasing in $h$ ). The next result (Proposition 3) shows that $\Pi_{C}^{*}>\Pi^{*}$ for any fixed $h$, thus we know that $\bar{h}_{C}>h^{\prime}$. Then $\bar{h} \leq h^{\prime}$ implies that $\bar{h}_{C}>\bar{h}$.

Proof of Proposition 3 First, from (1) and (7), we know that $q_{C}^{*}>q^{*}$ because $p^{*}<v$, which can be inferred from (2). Next, since $q_{C}^{*}>q^{*}$, this then implies that $p_{C}^{*}>p^{*}$, which follows from comparing (2) and (8), and the fact that $A(q)$ is increasing. Finally, we show that $\Pi_{C}^{*}>\Pi^{*}$ by comparing (3) and (9): this follows from observing that $q_{C}^{*}=\bar{F}^{-1}(c / v)$ is the maximizer of $\Pi(q)=v E(X \wedge q)-c q$. This completes the proof.

Proof of Proposition 4 Following our discussion in the text, the consumer's reservation price $r$ satisfies (10), and the seller's quantity $q$ maximizes the profit function given in (11). Together with consistency conditions $\xi_{\text {prob }}=A(q), \xi_{r}=r$, and the seller pricing at his belief of consumer reservation price $p=\xi_{r}$, we obtain the equilibrium conditions (12)-(13). Finally, to see (14), we have

$$
\begin{aligned}
\Pi_{G}^{*}=\Pi\left(p_{G}^{*}, q_{G}^{*}\right) & =p_{G}^{*} E\left(X \wedge q_{G}^{*}\right)-c q_{G}^{*}-w\left[E(X)-E\left(X \wedge q_{G}^{*}\right)\right] \\
& =\left(v-u-\frac{h-u}{A\left(q_{G}^{*}\right)}\right) E\left(X \wedge q_{G}^{*}\right)-c q_{G}^{*}-w\left[E(X)-E\left(X \wedge q_{G}^{*}\right)\right] \\
& =(v-u) E\left(X \wedge q_{G}^{*}\right)-c q_{G}^{*}-(h-u) E(X)-w\left[E(X)-E\left(X \wedge q_{G}^{*}\right)\right] \\
& =v E\left(X \wedge q_{G}^{*}\right)-c q_{G}^{*}-h E(X)+(u-w)\left[E(X)-E\left(X \wedge q_{G}^{*}\right)\right] .
\end{aligned}
$$

Next we consider the threshold search cost for existence of equilibrium when $w=u$, i.e., the boundary between efficient and inefficient guarantees. The equilibrium conditions (12)-(13) lead to

$$
\left(v-\frac{c}{\bar{F}(q)}\right) A(q)=h-u
$$

Recall that we have defined $J(q)=\left(v-\frac{c}{\bar{F}(q)}\right) A(q)$ in the proof of Proposition 1 and shown that it is concave in $q$. So we need $h \leq J(\hat{q})+u$ for an equilibrium solution to exist, where $\hat{q}$ is the 
maximizer of $J(q)$. Plugging $w=u$ into the seller's profit gives

$$
\Pi_{G}^{*}=v E\left(X \wedge q_{G}^{*}\right)-c q_{G}^{*}-h E(X),
$$

where $q_{G}^{*}$ satisfies (36). Note that the seller's profit function does not contain $u$. However, the compensation level $u$ affects the equilibrium quantity chosen by the seller. From the proof of Proposition 1, the seller's profit increases in $q_{G}^{*}$ for $q_{G}^{*}<\tilde{q}$ (recall $\bar{F}(\tilde{q})=\frac{c}{v}$ ), so again the seller will choose the larger root of (36) in equilibrium. By comparing (31) and (36), we know that $q^{*}<q_{G}^{*}<\tilde{q}$, and consequently $\Pi_{G}^{*}>\Pi^{*}$ (where $q^{*}$ and $\Pi^{*}$ are the equilibrium quantity and profit in the basic model without stockout compensation, respectively). Define $h^{\prime \prime}$ as the threshold such that $\Pi_{G}^{*}=0$, then we know $h^{\prime \prime}>h^{\prime}$, where $h^{\prime}$ is defined in the proof of Proposition 1. In addition, there is $h^{\prime \prime} \leq \bar{h}_{C}$ by comparing profit functions in (37) and (9).

Let $\bar{h}_{G}=(J(\hat{q})+u) \wedge h^{\prime \prime}$, then an equilibrium exists with the monetary compensation if and only if $h<\bar{h}_{G}$, where $\bar{h}_{C} \geq \bar{h}_{G}>\bar{h}=J(\hat{q}) \wedge h^{\prime}$. This completes the proof.

Proof of Proposition 5 The equilibrium quantity $q_{M}^{*}$ is determined by (36), where $J(q)=$ $\left(v-\frac{c}{\bar{F}(q)}\right) A(q)$ is concave in $q$. Since the seller will choose the larger root of (36) in equilibrium, we know that $q_{M}^{*}$ is increasing in $u$. This further implies that the seller's profit increases in $u$ because $\Pi_{M}^{*}=v E\left(X \wedge q_{M}^{*}\right)-c q_{M}^{*}-h E(X)$ is increasing in $q_{M}^{*}$. Manipulating equation (13) gives

$$
p_{M}^{*}=v-\frac{h-u}{A\left(q_{M}^{*}\right)}-u .
$$

This implies that $p_{M}^{*}$ is also increasing in $u$ because $h \geq u, A(q)$ increases in $u$ and $A(q)<1$.

Proof of Proposition 6 From the equilibrium condition (13), we see that when $u=h$, we must have $p_{M}^{*}=v-h$. In contrast, from the equilibrium condition (8) for the commitment case, we see that $p_{C}^{*}<v-h$ since $A\left(q_{C}^{*}\right)<1$. Next, observe that from $(12)$, we have $q_{M}^{*}=\bar{F}^{-1}(c / v)$ since $p_{M}^{*}+u=(v-h)+h=v$; similarly, we have $q_{C}^{*}=\bar{F}^{-1}(c / v)$ from (7). The final result that $\Pi_{M}^{*}=\Pi_{C}^{*}$ follows from comparing (9) and (14) and recalling that $q_{M}^{*}=q_{C}^{*}$.

Proof of Proposition 7 From the equilibrium condition (13), we see that with maximal compensation $u=h$, we must have $p_{G}^{*}=v-h$. In contrast, from the equilibrium condition (8) for the commitment case, we see that $p_{C}^{*}<v-h$ since $A\left(q_{C}^{*}\right)<1$. Next, observe that from (12), we have $q_{G}^{*}=\bar{F}^{-1}\left(\frac{c}{p_{G}^{*}+w}\right) \leq \bar{F}^{-1}(c / v)$ since $p_{G}^{*}+w \leq p_{G}^{*}+u=(v-h)+h=v$; in contrast, we have $q_{C}^{*}=\bar{F}^{-1}(c / v)$ from $(7)$. The final result that $\Pi_{G}^{*} \geq \Pi_{C}^{*}$ follows from Lemma 2 below. 
Lemma 1 Consider $\left(u_{1}, w_{1}\right)$ and $\left(u_{2}, w_{2}\right)$ with $u_{2}=u_{1}+\epsilon$ and $w_{2}=w_{1}+\epsilon$, where $\epsilon>0$. Then, in RE equilibrium, the seller's profit is higher under $\left(u_{2}, w_{2}\right)$ than $\left(u_{1}, w_{1}\right)$.

Proof of Lemma 1 Let $\Pi_{i}^{*}, q_{i}^{*}, p_{i}^{*}$ denote the RE equilibrium profit, quantity, and price under $\left(u_{i}, w_{i}\right)$ for $i=1,2$. Then, from $(14)$, we have

$$
\begin{aligned}
\Pi_{1}^{*} & =v E\left(X \wedge q_{1}^{*}\right)-c q_{1}^{*}-h E(X)+\left(u_{1}-w_{1}\right)\left[E(X)-E\left(X \wedge q_{1}^{*}\right)\right] \\
& =v E\left(X \wedge q_{1}^{*}\right)-c q_{1}^{*}-h E(X)+\left(u_{2}-w_{2}\right)\left[E(X)-E\left(X \wedge q_{1}^{*}\right)\right] \\
& \leq v E\left(X \wedge q_{2}^{*}\right)-c q_{2}^{*}-h E(X)+\left(u_{2}-w_{2}\right)\left[E(X)-E\left(X \wedge q_{2}^{*}\right)\right] \\
& =\Pi_{2}^{*}
\end{aligned}
$$

where it remains to show (40).

Consider the newsvendor function $\Pi(z)=v E(X \wedge z)-c z-\left(u_{2}-w_{2}\right) E(X \wedge z)$, which is maximized at $z^{*}=\bar{F}^{-1}\left(\frac{c}{v-\left(u_{2}-w_{2}\right)}\right)$, so $\Pi(z)$ is increasing for $z \leq z^{*}$. To establish (40), it suffices to show $q_{1}^{*} \leq q_{2}^{*} \leq z^{*}$.

We now prove the first inequality $q_{1}^{*} \leq q_{2}^{*}$ above. Let $\delta=u_{1}-w_{1}=u_{2}-w_{2}$. Manipulating (12) and (13) yields $\left(v-\delta-\frac{c}{\bar{F}\left(q_{i}^{*}\right)}\right) A\left(q_{i}^{*}\right)=h-u_{i}$. Recall from the proof of Proposition 1 that the function $J(q)=\left(v-\delta-\frac{c}{\bar{F}(q)}\right) A(q)$ is concave in $q$, starts at zero when $q=0$, increases and then decreases to $-\infty$. Since the seller chooses the larger solution of $J(q)=h-u_{i}$ as $q_{i}^{*}$, we conclude that with $u_{2} \geq u_{1}$, we must have $q_{2}^{*} \geq q_{1}^{*}$.

Finally, we show the second inequality $q_{2}^{*} \leq z^{*}$. Observe that $p_{2}^{*}+w_{2}=v-\left(u_{2}-w_{2}\right)-$ $\frac{h-u_{2}}{A\left(q_{2}^{*}\right)} \leq v-\left(u_{2}-w_{2}\right)$, since $u_{2} \leq h$. Therefore, the equilibrium quantity $q_{2}^{*}=\bar{F}^{-1}\left(\frac{c}{p_{2}^{*}+w_{2}}\right) \leq$ $\bar{F}^{-1}\left(\frac{c}{v-\left(u_{2}-w_{2}\right)}\right)=z^{*}$. This concludes the proof.

Lemma 2 Suppose the seller offers availability guarantee with $u=h$. Then, in RE equilibrium, as $w$ decreases, the seller's profit increases.

Proof of Lemma 2 Let $u=h$ and let $\Pi^{*}(w), q^{*}(w), p^{*}(w)$ denote the RE equilibrium profit, quantity, price corresponding to $w$. We have

$$
\begin{aligned}
\Pi^{*}(w) & =v E\left(X \wedge q^{*}(w)\right)-c q^{*}(w)-h E X+(u-w)\left[E X-E\left(X \wedge q^{*}(w)\right)\right] \\
& =\int_{0}^{q^{*}(w)}(v \bar{F}(q)-c) d q-h E X+(u-w) \int_{q^{*}(w)}^{\infty} \bar{F}(q) d q
\end{aligned}
$$


Therefore, for $\epsilon>0$, we have

$$
\begin{aligned}
\Pi^{*}(w+\epsilon)-\Pi^{*}(w) & =\int_{q^{*}(w)}^{q^{*}(w+\epsilon)}(v \bar{F}(q)-c) d q-(u-w) \int_{q^{*}(w)}^{q^{*}(w+\epsilon)} \bar{F}(q) d q-\epsilon \int_{q^{*}(w+\epsilon)}^{\infty} \bar{F}(q) d q(4) \\
& \leq \int_{q^{*}(w)}^{q^{*}(w+\epsilon)}[(v-(u-w)) \bar{F}(q)-c] d q \\
& \leq 0
\end{aligned}
$$

where the final inequality follows because $\bar{F}\left(q^{*}(w)\right)=\frac{c}{p^{*}(w)+w}=\frac{c}{v-h+w}$ and thus $(v-(u-w)) \bar{F}(q)-$ $c \geq 0$ if and only if $q \geq q^{*}(w)$, so, in particular, $(v-(u-w)) \bar{F}(q)-c \leq 0$ for every $q \in$ $\left[q^{*}(w), q^{*}(w+\epsilon)\right]$. This completes the proof.

Proof of Proposition 8 This result follows from Lemmas 1 and 2. Lemma 1 eliminates all $(u, w)$ combinations other than the points on curve $\mathrm{AB}$ (see Figure 1) and the vertical segment above point $\mathrm{B}$, which is then eliminated by Lemma 2. The points on curve $\mathrm{AB}$ are the only combinations satisfying conditions (i) and (ii) in the proposition.

Proof of Proposition 9 For any $q$, the value of $(u, w)$ that maximizes social welfare is the efficient level $\left(u^{* *}, w^{* *}\right)$, so this gives $(17)$. Now, with $\left(u^{* *}, w^{* *}\right)$, the social welfare function in terms of $q$ is a newsvendor profit function. The standard critical fractile solution yields (16).

Proof of Proposition 10 The results that $q_{B}^{*}=q^{F B}$ and $\left(u_{B}^{*}, w_{B}^{*}\right)=\left(u^{F B}, w^{F B}\right)$ has already been established in the discussion preceding the proposition. Next, by rearranging equilibrium condition (18), we obtain $p_{B}^{*}=\left(v-u^{F B}-\frac{h-u^{F B}}{A\left(q^{F B}\right)}\right)$. It also follows from (18) that $\left(v-p_{B}^{*}\right) A\left(q_{B}^{*}\right)=$ $h-u_{B}^{*} \bar{A}\left(q_{B}^{*}\right) \geq h A\left(q_{B}^{*}\right)$, since $u_{B}^{*} \leq h$, and this then implies that $v-p_{B}^{*} \geq h$ as desired. Finally, the seller extracts the entire social surplus because we have already seen that from (22) that his profit function is equivalent to the social welfare function, which implies that consumer surplus is zero.

Proof of Proposition 11 In the dynamic program $(24)$, consider the optimal action $q^{*}(\hat{\xi})$ in state $\hat{\xi} \in[0,1]$. Note that when $\hat{\xi}=\epsilon \approx 0$, we have $q^{*}(\hat{\xi})=0$ and thus $A\left(q^{*}(\hat{\xi})\right)=0$, and when $\hat{\xi}=1-\epsilon$, we have $A\left(q^{*}(\hat{\xi})\right)<\hat{\xi}$. Therefore, as $\hat{\xi}$ increases from 0 to $1, A\left(q^{*}(\hat{\xi})\right)$ first starts below $\hat{\xi}$ and then ends below $\hat{\xi}$.

Suppose the curve of $A\left(q^{*}(\hat{\xi})\right)$ never intersects the curve of $\hat{\xi}$. In other words, $A\left(q^{*}(\hat{\xi})\right)<\hat{\xi}$ for every $\hat{\xi} \in(0,1]$. In this case, for all initial beliefs $\hat{\xi}$, we have $q_{t}^{*} \rightarrow 0$ and $\hat{\xi}_{t} \rightarrow 0$, so the proposition 
holds with $z=1$.

Next, suppose that $A\left(q^{*}(\hat{\xi})\right)=\hat{\xi}$ at some $\hat{\xi}=\dot{\xi}$. By the optimality of $q^{*}(\dot{\xi})$ at state $\dot{\xi}$, we have the following first order condition at $q=q^{*}(\dot{\xi})$ and $\hat{\xi}=\dot{\xi}$ :

$$
\begin{aligned}
\frac{\partial}{\partial q} \Pi(q, \hat{\xi})+\delta V^{\prime}(\alpha A(q)+\bar{\alpha} \hat{\xi}) \cdot \alpha A^{\prime}(q) & =0 \\
\frac{\partial}{\partial q} \Pi(q, \hat{\xi})+\delta V^{\prime}(\hat{\xi}) \cdot \alpha A^{\prime}(q) & =0 .
\end{aligned}
$$

Using the envelope theorem on Bellman equation (24) we know that

$$
V^{\prime}(\hat{\xi})=\frac{\partial}{\partial \hat{\xi}} \Pi\left(q^{*}(\hat{\xi}), \hat{\xi}\right)+\bar{\alpha} \delta V^{\prime}\left(\alpha A\left(q^{*}(\hat{\xi})\right)+\bar{\alpha} \hat{\xi}\right),
$$

and in particular, at $\hat{\xi}=\dot{\xi}$, this reduces to

$$
V^{\prime}(\hat{\xi})=\frac{1}{1-\bar{\alpha} \delta} \cdot \frac{\partial}{\partial \hat{\xi}} \Pi\left(q^{*}(\hat{\xi}), \hat{\xi}\right) .
$$

Therefore, at $q=q^{*}(\dot{\xi})$ and $\hat{\xi}=\dot{\xi}$, the first order condition in (47) above becomes

$$
\frac{\partial}{\partial q} \Pi(q, \hat{\xi})+\frac{\alpha \delta}{1-\bar{\alpha} \delta} \cdot A^{\prime}(q) \cdot \frac{\partial}{\partial \hat{\xi}} \Pi(q, \hat{\xi})=0 .
$$

When $\delta=0$, this condition becomes $\frac{\partial}{\partial q} \Pi(q, \hat{\xi})=(v-h / \hat{\xi}) \bar{F}(q)-c=0$. Together with the requirement that $A(q)=\hat{\xi}$ imposed above, these are precisely the conditions for an RE equilibrium in Proposition 1. Specifically, we have $(v-h / A(q)) \bar{F}(q)-c=0$. Since we established that there are exactly two solutions to this equations, there must also be exactly two such $\hat{\xi}$ for which $A\left(q^{*}(\hat{\xi})\right)=\hat{\xi}$. Finally, when $\delta \in(0,1)$, condition $(48)$, together with $A(q)=\hat{\xi}$, becomes

$$
\begin{aligned}
& \left(v-\frac{h}{A(q)}\right) \bar{F}(q)-c+\frac{\alpha \delta}{1-\bar{\alpha} \delta} \cdot \frac{\bar{F}(q)}{E(X)} \cdot \frac{h}{A(q)^{2}} E(X \wedge q)=0, \\
& \left(v-\frac{h}{A(q)}\right) \bar{F}(q)-c+\frac{\alpha \delta}{1-\bar{\alpha} \delta} \cdot \bar{F}(q) \cdot \frac{h}{A(q)}=0, \\
& \left(v-\left(1-\frac{\alpha \delta}{1-\bar{\alpha} \delta}\right) \frac{h}{A(q)}\right) \bar{F}(q)-c=0 .
\end{aligned}
$$

Replacing $h$ with $\left(1-\frac{\alpha \delta}{1-\bar{\alpha} \delta}\right) h=\left(\frac{1-\delta}{1-\bar{\alpha} \delta}\right) h$, note that the preceding equation also has exactly two solutions.

Finally, since there are two points where the curve of $A\left(q^{*}(\hat{\xi})\right)$ intersects $\hat{\xi}$, the curve must first start below $\hat{\xi}$, intersect and rise above $\hat{\xi}$, and then intersect and fall below $\hat{\xi}$. Let the two points be $y_{1}$ and $y_{2}$. Then, when initial beliefs start below $y_{1}$ they will converge to zero, but when initial beliefs start above $y_{1}$ they will converge to $y_{2}$. This proves our result by taking $z=y_{1}$ and $\widetilde{\xi}=y_{2}$. 
Proof of Proposition 12 From the proof of Proposition 1, recall that $q^{*}[h]$ is the larger solution of $(v-h / A(q)) \bar{F}(q)-c=0$. Note that when we replace $h$ by $\frac{1-\delta}{1-(1-\alpha) \delta} \cdot h$, we get precisely equation (25) in Proposition 11(ii). This proves our result.

Proof of Proposition 13 The seller's problem can be expressed as

$$
\begin{aligned}
\max _{p} \pi= & \sum_{i} \lambda_{i}\left[p E\left(X \wedge \hat{Q}_{i}(p)\right)-c \hat{Q}_{i}(p)\right] \\
& \text { s.t. } \sum_{i} \lambda_{i j}(v-p) \xi_{i}\left(\hat{Q}_{i}(p)\right)-h=0, j=1, \ldots, M .
\end{aligned}
$$

Since the effective quantity $\hat{Q}_{i}=Q_{i} / \lambda_{i}$ satisfies $\left.F \hat{Q}_{i}\right)=(p-c) / p$ in the RE equilibrium for all $i$, we know that the seller will choose the same effective quantity, denoted by $\hat{Q}(p)$, for all product variants. Note also that $\sum_{i} \lambda_{i j}=1$ for all $j$ and $\sum_{i} \lambda_{i}=1$. Thus the seller's problem can be rewritten as

$$
\begin{aligned}
\max _{p} \pi= & p E(X \wedge \hat{Q}(p))-c \hat{Q}(p) \\
& \text { s.t. }(v-p) A(\hat{Q}(p))-h=0
\end{aligned}
$$

From the seller's point of view, the problem is to set a price $p$ for all products to induce customers to visit the store, and the consumers' search cost is $h$. This implies that the multi-product problem can be viewed as $N$ independent single-product problems with identical structure. Therefore, the $\mathrm{RE}$ equilibrium for the multi-product model can be characterized using the same set of conditions for the single-product model.

Proof of Proposition 14 The following condition holds for the marginal consumer $\theta$ in any RE equilibrium in the heterogeneous model:

$$
\left(v_{\theta}-p\right) A\left(q / s\left(v_{\theta}\right)\right)+u\left(1-A\left(q / s\left(v_{\theta}\right)\right)\right)=h .
$$

That is, the marginal consumer's expected utility from visiting the seller should be equal to zero (and therefore the consumer is indifferent between visiting and not visiting the seller). The seller's expected profit is $\Pi=(p+w) E\left(s\left(v_{\theta}\right) X \wedge q\right)-c q-w E\left(s\left(v_{\theta}\right) X\right)$. Given the market base $s\left(v_{\theta}\right)$ and price $p$, the seller will choose the profit-maximizing $q$ given by

$$
F\left(q / s\left(v_{\theta}\right)\right)=\frac{p+w-c}{p+w} .
$$


Under the notation $z=q / s\left(v_{\theta}\right)$, we can express $p$ and $v_{\theta}$ in terms of $z$ as

$$
\begin{aligned}
p & =\frac{c}{\bar{F}(z)}-w \\
v_{\theta} & =\frac{c}{\bar{F}(z)}+\frac{h-u}{A(z)} .
\end{aligned}
$$

The seller's profit can be written as a function of $z$ :

$$
\Pi(z)=s\left(v_{\theta}(z)\right)\left[\frac{c}{\bar{F}(z)} E(X \wedge z)-c z-w E(X)\right] .
$$

Note that (49) and (50) are necessary in any RE equilibrium. Since the seller's profit can be expressed as a function of $z$ (equation 51), we may search for RE equilibrium consisting of $p, z$, and $v_{\theta}$ (this is equivalent to $p, q$, and $v_{\theta}$ because $q=s\left(v_{\theta}\right) z$ ). For any equilibrium to exist, we need to satisfy the following three conditions:

(i) There must be $v_{\theta}<v$, which implies $v_{\theta}(z)=\frac{c}{\bar{F}(z)}+\frac{h-u}{A(z)}<v$ for some $z$. (We ignore the trivial equilibrium in which $v_{\theta}=v$ and the seller attracts zero demand.) Taking derivative of $v_{\theta}(z)$ gives

$$
v_{\theta}^{\prime}(z)=\frac{c}{\bar{F}(z)} \frac{f(z)}{\bar{F}(z)}-\frac{(h-u) \bar{F}(z)}{A^{2}(z) E(X)} .
$$

For $u=h$, it is clear that $v_{\theta}(z)=\frac{c}{\bar{F}(z)}$ increases from $c$ to infinity as $z$ increases. Thus $v_{\theta}(z)<v$ for some $z$. For $u<h$, since $F$ has an increasing failure rate and $A(z)$ is increasing in $z$, we know that $v_{\theta}^{\prime}(z)<0$ first and then $v_{\theta}^{\prime}(z)>0$. That is, $v_{\theta}(z)$ decreases first and then increases in $z$, with a unique minimizer. Let $\hat{z}$ be the unique minimizer. Applying the Envelope Theorem gives $\frac{d v_{\theta}(\hat{z})}{d(h-u)}=\frac{1}{A(\hat{z})}>0$, meaning that $v_{\theta}(\hat{z})$ is increasing in $h-u$. Let $\bar{h}_{H} \in(0, h]$ be such that $v_{\theta}(\hat{z})=v$ when $h-u=\bar{h}_{H}$. (Since $v_{\theta}(\hat{z})<v$ for $h-u=0$, we know $\bar{h}_{H}>0$. If $v_{\theta}(\hat{z})<v$ even for $h-u=h$, then set $\bar{h}_{H}=h$.) Thus $v_{\theta}(\hat{z})<v$ only if $h-u<\bar{h}_{H}$. That is, if $h-u \leq \bar{h}_{H}$, there does not exist a $z$ such that $v_{\theta}<v$. In this case, only non-participatory equilibrium may exist (i.e., the seller stocks nothing and attracts zero demand). On the other hand, if $h-u<\bar{h}_{H}$, then $v_{\theta}(z)=v$ has two solutions. We may use $z_{1}$ and $z_{2}\left(z_{1}<z_{2}\right)$ to denote the solutions. Then, there is $v_{\theta}<v$ for all $z: z_{1}<z<z_{2}$.

(ii) The seller's equilibrium profit in (51) should be positive. Note that $s\left(v_{\theta}(z)\right)>0$ for all $z$. Thus we need $K(z)=\frac{c}{\bar{F}(z)} E(X \wedge z)-c z-u E(X)>0$. Taking derivatives of $K(z)$ yields

$$
K^{\prime}(z)=\frac{c f}{\bar{F}^{2}(z)} E(X \wedge z) \geq 0 \text { and } K^{\prime \prime}(z)>0,
$$

where $K^{\prime \prime}(z)>0$ follows from the fact that $F$ has an increasing failure rate. Thus $K(z)=0$ has a unique solution $z_{3}>0$ such that $K(z)>0$ if and only $z>z_{3}$. 
(iii) Finally, we need to ensure that the marginal consumer $\theta$ should be willing to buy the product, i.e., $v_{\theta} \geq p$. Otherwise if $v_{\theta}<p$, then the consumer will visit the seller but never purchase the product, which only incurs costs to the seller. This cannot be sustained in equilibrium. However, $v_{\theta} \geq p$ is equivalent to $u+\frac{h-u}{A(z)} \geq 0$, which clearly holds since $u \leq h$.

Combining the above conditions we know that an equilibrium exists if and only if $h-u<\bar{h}_{H}$ and $z_{2}>z_{3}$.

Proof of Proposition 15 The seller's profit with commitment can be written as

$$
\begin{aligned}
\Pi(u, w ; p, z)= & s\left(v_{\theta}\right)[(p+w) E(X \wedge z)-c z-w E(X)] \\
= & s\left(p-u\left(\frac{1}{A(z)}-1\right)+\frac{h}{A(z)}\right) \\
& \cdot\left[p-u\left(\frac{1}{A(z)}-1\right)+(u-w)\left(\frac{1}{A(z)}-1\right)-\frac{c z}{E(X \wedge z)] E(X \wedge z) .}\right.
\end{aligned}
$$

Note that $\frac{1}{A(z)}-1>0$ since $A(z)<1$. Suppose $\left(u^{\prime}, w^{\prime} ; p^{\prime}, z^{\prime}\right)$ represent the seller's optimal guarantee format and corresponding decisions with $u^{\prime}-w^{\prime}<u^{* *}-w^{* *}$. Next we show that the seller's profit $\Pi\left(u^{\prime}, w^{\prime} ; p^{\prime}, z^{\prime}\right)$ can be further improved. Consider $\left(u^{* *}, w^{* *} ; p^{*}, z^{*}\right)$, where

$$
z^{*}=z^{\prime} \text { and } p^{*}+u^{* *}\left(\frac{1}{A\left(z^{*}\right)}-1\right)=p^{\prime}+u^{\prime}\left(\frac{1}{A\left(z^{\prime}\right)}-1\right) .
$$

It is clear that $\Pi\left(u^{* *}, w^{* *} ; p^{*}, z^{*}\right)>\Pi\left(u^{\prime}, w^{\prime} ; p^{\prime}, z^{\prime}\right)$ since $u^{* *}-w^{* *}>u^{\prime}-w^{\prime}$. Therefore, the seller's optimal guarantee format is $u^{*}=u^{* *}$ and $w^{*}=w^{* *}=\underline{W}\left(u^{*}\right)$. 